University of Warwick institutional repository: http://go.warwick.ac.uk/wrap This paper is made available online in accordance with publisher policies. Please scroll down to view the document itself. Please refer to the repository record for this item and our policy information available from the repository home page for further information.

To see the final version of this paper please visit the publisher's website. Access to the published version may require a subscription.

Author(s): A. J. COOPER and N. PEAKE

Article Title: Upstream-radiated rotor-stator interaction noise in mean swirling flow

Year of publication: 2005

Link to published

version: http://dx.doi.org/10.1017/S0022112004002010

Publisher statement: None 


\title{
Upstream-radiated rotor-stator interaction noise in mean swirling flow
}

\author{
By A. J. COOPER ${ }^{1}$ AND N. PEAKE P $^{2}$ \\ ${ }^{1}$ School of Engineering, University of Warwick, Gibbet Hill Road, Coventry CV4 7AL, UK \\ ${ }^{2}$ Department of Applied Mathematics and Theoretical Physics, University of Cambridge, \\ Centre for Mathematical Sciences, Wilberforce Road, Cambridge CB3 0WA, UK
}

(Received 17 June 2003 and in revised form 30 April 2004)

A major component of the noise in modern aeroengines is rotor-stator interaction noise generated when the wake from the rotating fan impinges on a stator row downstream. An analytically based model for the prediction of upstream-radiated rotor-stator interaction noise is described, and includes the important effect of mean swirling flow on both the rotor wake evolution and the acoustic response. The analytic nature of the model allows for the inclusion of all wake harmonics and enables the response at all blade passing frequencies to be determined.

An asymptotic analysis based on large rotor blade number is used to model the evolution of the rotor wake downstream in a cylindrical duct carrying mean swirling flow. The equations governing the axial evolution of the wake simplify to three coupled first-order differential equations in the interior, while close to the duct walls, a boundary-layer correction is required in order to satisfy the impermeability conditions at the boundaries. At the stator location, the wake is used as input into a local linear cascade model at each radius. The interaction of each wake harmonic gives rise to acoustic waves of multiple azimuthal order which contribute to the pressure field radiated back upstream. This enables the total acoustic response to be determined in terms of cylindrical duct modes in mean swirling flow.

The effect of stator blade geometry (thickness, camber, angle of attack) and rotorstator separation on the total upstream-radiated noise is determined. Blade geometry is shown to have a significant effect on the noise generated, and increasing the rotorstator gap can lead to large reductions in noise levels. Asymptotic treatment of the acoustic field, based on large azimuthal order, is also considered and used to identify the dominant contributions to the total pressure field resulting from the rotor-stator interaction. The ray structure of the acoustic modes in swirl is shown to be very different in some cases from that in uniform flow.

\section{Introduction}

Noise reduction is now one of the major issues in aeroengine design. In modern highbypass ratio turbofan systems much of the noise is generated by the fan, particularly by the interaction between the fan-blade wakes and downstream vanes, as well as by rotor-stator interactions in the compressor. At present, however, methods for predicting the noise resulting from these interactions are of limited scope. Here, a new analytically based method is developed which models important features such as the three-dimensional duct geometry, swirling flow behind the fan, and statorblade geometry such as thickness and camber distributions and inclination to the 
oncoming flow. Analytical models of this type allow significant insight into the physical mechanisms behind the noise-generation process and have practical value as an efficient quantitative prediction scheme.

Previous analytical approaches to determining the sound radiation produced by wake-stator interactions have considered the two-dimensional problem obtained by unwrapping the duct at a fixed radius so that the stator becomes a linear cascade of blades. Analyses in this type of framework have been carried out by Mani \& Horvay (1970), Koch (1971) and Peake (1992) using the Wiener-Hopf technique to consider a single incident gust convected by a uniform flow. One obvious drawback of this approach is that the three-dimensional effects of the cylindrical geometry and the effects of swirl on the wake evolution cannot be captured. In this paper, the approach will be to exploit the large blade number typical of modern systems so as to allow the effects of swirl to be included, and to allow a better representation of the three-dimensional geometry.

The general approach will be to determine the wake evolution downstream of the fan in mean swirling flow using an asymptotic model based on large fan blade number, and combine this with the cascade approach to determine the upstream noise in the duct generated by the interaction of this wake with a downstream stator. The model is therefore based on a quasi-three-dimensional approach which accounts for the radial variation of the incident gust and reconstructs the resulting sound field in the three-dimensional annular duct. The evolved wake will be fed locally into the cascade model of Evers \& Peake (2002) at each radius along the span. This cascade model allows the effects of airfoil thickness and camber to be determined, and builds on the work of Myers \& Kerschen $(1995,1997)$ and Tsai \& Kerschen (1990) who developed an asymptotic analysis in the limit of large reduced frequency for the case of a single airfoil, and Peake \& Kerschen (1997) who predicted the noise generated by a cascade of flat plates at non-zero angle of attack. The influence of swirl on the local cascade response is included systematically. The asymptotic cascade calculation is able to predict the upstream sound radiation (in terms of plane-wave modes) produced by a single incident gust. In this paper, cascade calculations will be used to provide the acoustic pressure field across the whole duct annulus just upstream of the stator. This pressure data is then used as a boundary condition to determine the amplitudes of the acoustic duct modes propagating upstream from the stator.

Centrifugal and Coriolis forces induced by the mean swirl prevent any vortical disturbances from being purely convected. Instead, they are 'nearly' convected and undergo significant spatial distortion as they move downstream. The governing equations for the unsteady vorticity and pressure are coupled by the presence of mean vorticity and give rise to coupled acoustic-vorticity waves. Two families exist, one which is pressure-dominated (nearly sonic) and well-defined by a modal decomposition, and a second which is vorticity-dominated (nearly convected). Golubev \& Atassi $(1996,1998)$ showed that, for the vorticity-dominated waves, a critical layer exists in the eigenmode spectrum, either side of which the eigenmodes corresponding to the nearly convected modes cluster. As such, it is not generally appropriate to describe a vortical disturbance in terms of modes, but instead as the solution to an initial-value problem (Golubev \& Atassi 2000a). The full system of equations describing the initial-value problem consists of four coupled partial differential equations. However, using our asymptotic analysis, only dominant terms in the equations are retained; this reduces the system to three coupled first-order differential equations in the flow interior, plus a boundary layer on the walls. This 
considerable simplification provides a very efficient method of computation for the wake evolution.

Swirling flow can also have a significant effect on the propagation of sound, and it is crucial to relate the sound waves predicted in the cascade formulation to sound waves in annular-duct swirling flow. The presence of mean swirl can cut on or cut off modes according to the sense of rotation with respect to the mean swirl. Modes which are cut on in a non-swirling flow can be cut off by the presence of mean swirl if they are co-rotating with the swirl (positive azimuthal order) and those which are cut off in the absence of swirl may be cut on in the presence of mean swirl if they rotate in the opposite sense to the mean swirl (negative azimuthal order). This becomes important in the rotor-stator interaction problem where a single incident harmonic wave can be reflected into acoustic modes of multiple azimuthal order (both positive and negative). Asymptotic analysis of the acoustic field in swirling flow based on large azimuthal order is also considered and used to determine rays paths in the duct and to determine dominant contributions to the radiated sound field.

The linear cascade approximation at each radius has been used by Glegg (1999) and Hanson (2001) to determine the scattered acoustics in a duct for the case of unloaded blades and without accounting for the effects of swirl. Large bladenumber, or high-frequency, asymptotics have been applied to aspects of this problem previously. Envia (1998) used a high-radial-order analysis to represent the duct acoustic field in uniform flow. Golubev \& Atassi (2000b) noted that the coupling between the potential and vortical parts of the unsteady velocity in mean swirling flow is weak at high frequencies. This led to an asymptotic expansion for the pressuredominated modes in powers of $1 / B$, with $B$ the number of fan blades, so that the vortical part could be neglected to leading order. Elhadidi \& Atassi (2002, 2003) have also exploited this weak coupling at high frequencies to investigate the effects of mean swirl and blade loading on interaction noise in annular ducts. In particular, they have found that unsteady blade loading, the number of propagating modes and sound pressure levels are dependent strongly on swirl and frequency, underlining the importance of including swirl in any rotor-stator interaction noiseprediction scheme. In this paper, a full asymptotic analysis is carried out to further simplify the equations in this asymptotic limit. A numerical approach to the scattering of disturbances by an annular cascade in swirling flow has been carried out by Atassi et al. (2004).

The formulation of the model is presented as follows. The asymptotic model for the wake evolution and sample results are presented in $\S 2$. The interaction of the fan wake with the cascade is described in $\S 3$. Sample results showing the effects of stator-blade geometry and rotor-stator separation on the resulting sound field are presented in $\S 4$. An asymptotic description of the acoustic field is given in $\S 5$ and the paper is summarized in $\S 6$.

\section{Asymptotic model for rotor-wake evolution}

\subsection{Evolution equations}

The region downstream of the rotor is taken to be an axisymmetric annulus with constant cross-section. Lengths are non-dimensionalized by the tip radius so that the duct is defined in the region $r_{h} \leqslant r \leqslant 1$. Velocities are non-dimensionalized with respect to the stagnation speed of sound $c_{0}^{*}$, and subsequently all quantities used are non-dimensional. 
The total velocity field downstream of the rotor is expressed in terms of a steady, axisymmetric mean flow and a small-amplitude unsteady perturbation

$$
\boldsymbol{U}_{t o t}(x, r, \theta, t)=\boldsymbol{U}(r)+\boldsymbol{u}^{\prime}(x, r, \theta, t),
$$

with $(x, r, \theta)$ cylindrical coordinates, the $x$-axis pointing downstream and $x=0$ taken to be the rotor trailing-edge plane. The steady mean flow is assumed to have the form

$$
\boldsymbol{U}=U(r) \boldsymbol{e}_{x}+W(r) \boldsymbol{e}_{\theta} .
$$

The mean vorticity, $\boldsymbol{\xi}$, for this mean flow profile is

$$
\boldsymbol{\xi}=\frac{1}{r} \frac{\partial(r W)}{\partial r} \boldsymbol{e}_{x}-\frac{\partial U}{\partial r} \boldsymbol{e}_{\theta} .
$$

The unsteady perturbation velocity is decomposed into vortical and potential parts, according to Goldstein (1978), such that

$$
\boldsymbol{u}^{\prime}=\boldsymbol{u}+\nabla \Phi
$$

with the perturbation pressure expressed only in terms of the potential by

$$
p^{\prime}=-\rho_{0} \frac{\mathrm{D} \Phi}{\mathrm{D} t}
$$

where $\mathrm{D} / \mathrm{D} t=\partial / \partial t+\boldsymbol{U} \cdot \nabla$ is the convective derivative, and $\rho_{0}(r)$ is the mean density.

Where the amplitude of the perturbation is small compared to the mean flow, the evolution of the unsteady disturbance is governed by the linearized Euler equations which, when the mean entropy is uniform, can be written in the form

$$
\frac{\mathrm{D} \boldsymbol{u}}{\mathrm{D} t}+(\boldsymbol{u} \cdot \nabla) \boldsymbol{U}=-\boldsymbol{\xi} \times \nabla \Phi
$$

while the continuity equation becomes

$$
\frac{\mathrm{D}}{\mathrm{D} t} \frac{1}{c_{0}^{2}} \frac{\mathrm{D}}{\mathrm{D} t} \Phi-\frac{1}{\rho_{0}} \nabla \cdot\left(\rho_{0} \nabla\right) \Phi=\frac{1}{\rho_{0}} \nabla \cdot\left(\rho_{0} \boldsymbol{u}\right),
$$

where $c_{0}(r)$ is the local speed of sound.

The wake shed from the rotor blades gives rise to a vortical disturbance which can be expressed as a superposition of harmonic waves. If the perturbation is expressed in terms of modes, then, as shown by Golubev \& Atassi (1996, 1998), the eigenmodes produced by a mean vortical velocity are coupled acoustic--vorticity modes. These modes are divided into two families, one of which is pressure-dominated and corresponds to nearly sonic modes, and another of which is vorticity dominated and corresponds to nearly convected modes. However, Golubev \& Atassi $(1996,1998)$ found that it is not practical to describe a vorticity-dominated disturbance using a modal decomposition because the eigenmodes cluster near the edges of a critical layer (with the centre corresponding to pure convection). This poses problems in resolving the eigenmodes with sufficient accuracy and with the calculation of the associated eigenvectors. Therefore, as in Golubev \& Atassi (2000a) and Elhadidi et al. (2000), it is more appropriate to determine the evolution of the vortical disturbance using an initial-value analysis, where the wake profile at the rotor blade trailing edges provides the initial condition for integration downstream.

The initial wake profile $f\left(r, \theta^{\prime}\right)$ (at $x=0$ ) is aligned with the fan blades in the rotating frame, where $\theta^{\prime}$ is the polar angle measured in the rotating frame, and appears as a velocity deficit behind each fan blade trailing edge. If the rotor has $B$ 
blades, then the wake profile can be Fourier-decomposed into harmonics which are integer multiples of the number of fan blades,

$$
f\left(r, \theta^{\prime}\right)=\sum_{N=-\infty}^{\infty} \beta_{N B}(r) \mathrm{e}^{\mathrm{i} N B \theta^{\prime}} .
$$

Typically, a fan has of the order of 20 rotor blades, so that the azimuthal mode number, $m=N B$ for integer $N$, can be treated as a large parameter.

If the fan rotates with a non-dimensional angular velocity $\Omega_{F}$ then the disturbance in the non-rotating frame is time dependent, and can be written in the form

$$
\begin{aligned}
\boldsymbol{u} & =\boldsymbol{a}(x, r) \mathrm{e}^{\mathrm{i} m k(r) x+\mathrm{i} m \theta-\mathrm{i} \omega t}, \\
\Phi & =\phi(x, r) \mathrm{e}^{\mathrm{i} m k(r) x+\mathrm{i} m \theta-\mathrm{i} \omega t},
\end{aligned}
$$

where the frequency $\omega=m \Omega_{F}$. The axial wavenumber is $m k(r)$ and the radial wavenumber is $m x k_{r} \equiv m x \partial k / \partial r$. Since the disturbance is not purely convected, the amplitudes depend on both radial and axial location.

The disturbance amplitudes are now expanded in terms of the large parameter $m$ as follows:

$$
\boldsymbol{a}=\boldsymbol{a}_{0}+\frac{\boldsymbol{a}_{1}}{m}+O\left(1 / m^{2}\right), \quad \phi=\phi_{0}+\frac{\phi_{1}}{m}+O\left(1 / m^{2}\right) .
$$

An asymptotic analysis is carried out on the governing equations, (6) and (7), retaining only the leading-order terms in each equation. Under these assumptions, (7) reduces to

$$
\left\{\frac{\Lambda^{2}}{c_{0}^{2}}-m^{2} x^{2} k_{r}^{2}-\frac{m^{2}}{r^{2}}-m^{2} k^{2}\right\}\left(\phi_{0}+\frac{\phi_{1}}{m}\right)=O(m),
$$

where $\Lambda=m k U-\omega+m W / r$. In order for (12) to hold for all values of $r$ and $x$, we must have

$$
\phi_{0}=0 \text {. }
$$

The leading-order terms from (6) are then

$$
\mathrm{i} \Lambda \boldsymbol{a}_{0}=O(1) .
$$

In order for terms to balance, assuming $\boldsymbol{a}_{0}$ is $O(1)$, we set $\Lambda=0$. This then defines the axial wavenumber as

$$
m k=(\omega-m W / r) / U
$$

which is the wavenumber of disturbances which are exactly convected. The evolution of the gust amplitudes is determined by the equations at the next order, which, using the notation $\boldsymbol{a}_{0}=\left(a_{x}, a_{r}, a_{\theta}\right)$, are

$$
\begin{aligned}
U \frac{\partial a_{x}}{\partial x}+\frac{\partial U}{\partial r} a_{r} & =-\frac{\partial U}{\partial r} x k_{r} \mathrm{i} \phi_{1}, \\
U \frac{\partial a_{r}}{\partial x}-2 \frac{W}{r} a_{\theta} & =\left\{\frac{\Gamma}{r}+k \frac{\partial U}{\partial r}\right\} \mathrm{i} \phi_{1}, \\
U \frac{\partial a_{\theta}}{\partial x}+\Gamma a_{r} & =-\Gamma x k_{r} \mathrm{i} \phi_{1}, \\
\left\{x^{2} k_{r}^{2}+\frac{1}{r^{2}}+k^{2}\right\} \phi_{1} & =\mathrm{i}\left\{x k_{r} a_{r}+\frac{1}{r} a_{\theta}+k a_{x}\right\},
\end{aligned}
$$

where $\Gamma=(1 / r) \partial(r W) / \partial r$. Since no $r$-derivatives appear in these equations, $r$ acts solely as a parameter. 
Substitution for $\phi_{1}$ from (19) into (16)-(18) reduces the equations to three coupled first-order differential equations which can be written in the form

$$
\frac{\partial \boldsymbol{a}_{0}}{\partial x}=\boldsymbol{M}(x, r) \boldsymbol{a}_{0},
$$

where $\boldsymbol{M}$ is a $3 \times 3$ matrix. These equations can be solved numerically given a set of initial conditions at $x=0$. The solution to (20), however, cannot satisfy the impermeability condition of zero normal velocity at the duct walls because the assumption that radial derivatives such as $\partial a_{r} / \partial r$ are $O(1)$ breaks down close to the boundaries. In order to overcome this, a boundary-layer correction, which is exponentially small at distances $O(1)$ away from the walls, can be derived which when superposed onto the existing solution enables the boundary conditions to be satisfied. This effectively defines a hydrodynamic boundary layer of thickness $O(1 / \mathrm{m})$ at each wall. In order to calculate this correction, a rescaling of the radius must be carried out, and the analysis for this is described in the next subsection.

\subsection{Boundary-layer correction}

A rescaling of the radius is carried out at each boundary to define

$$
R= \pm|m|\left\{r-r_{b}\right\} \in[0, \infty),
$$

where $r_{b}=r_{h}, r_{t}(=1)$, with the positive(negative) sign referring to the inner(outer) radius. With this rescaling

$$
\frac{\partial}{\partial r} \rightarrow \pm|m| \frac{\partial}{\partial R}
$$

The mean flow and axial wavenumber are expanded about the boundary radius $r_{b}$, and the boundary-layer correction takes the form

$$
\left[\hat{\boldsymbol{a}_{0}}, \hat{\phi}_{1} / m\right]_{r_{b}}(x, R) \exp \left(\mathrm{i} m\left\{k\left(r_{b}\right) x+\left(r-r_{b}\right) k_{r}\left(r_{b}\right) x\right\}+\mathrm{i} m \theta-\mathrm{i} \omega t\right),
$$

subject to the leading-order boundary conditions

$$
\begin{gathered}
\hat{\boldsymbol{a}}_{0}, \hat{\phi}_{1} \rightarrow 0 \text { as } R \rightarrow \infty, \\
a_{r}\left(x, r_{b}\right)+\hat{a}_{r}(x, 0)+\mathrm{i} x k_{r}\left(r_{b}\right)\left\{\phi_{1}\left(x, r_{b}\right)+\hat{\phi}_{1}(x, 0)\right\} \pm \frac{\partial \hat{\phi}_{1}}{\partial R}(x, 0)=0 .
\end{gathered}
$$

The leading-order equations governing the boundary-layer correction are, from (6) and (7),

$$
\begin{aligned}
& U \frac{\partial \hat{a}_{x}}{\partial x}+\frac{\partial U}{\partial r} \hat{a}_{r}=-\frac{\partial U}{\partial r}\left\{x k_{r} \mathrm{i} \hat{\phi}_{1} \pm \frac{\partial \hat{\phi}_{1}}{\partial R}\right\}, \\
& U \frac{\partial \hat{a}_{r}}{\partial x}-2 \frac{W}{r_{b}} \hat{a}_{\theta}=\left\{\frac{\Gamma}{r_{b}}+k \frac{\partial U}{\partial r}\right\} \mathrm{i} \hat{\phi}_{1}, \\
& U \frac{\partial \hat{a}_{\theta}}{\partial x}+\Gamma \hat{a}_{r}=-\Gamma\left\{x k_{r} \mathrm{i} \hat{\phi}_{1} \pm \frac{\partial \hat{\phi}_{1}}{\partial R}\right\}, \\
& \frac{\partial^{2} \hat{\phi}_{1}}{\partial R^{2}} \pm 2 \mathrm{i} x k_{r} \frac{\partial \hat{\phi}_{1}}{\partial R}-\lambda^{2} \hat{\phi}_{1}=\mp \frac{\partial \hat{a}_{r}}{\partial R}-\mathrm{i} x k_{r} \hat{a}_{r}-\frac{\mathrm{i}}{r} \hat{a}_{\theta}-\mathrm{i} k \hat{a}_{x},
\end{aligned}
$$

where $\lambda^{2}=x^{2} k_{r}^{2}+k^{2}+\left(1 / r^{2}\right)$ and $k$ and the mean flow terms are evaluated at $r=r_{b}$. Given that the initial wake will satisfy the impermeability condition, the initial conditions for the boundary-layer correction are $\hat{\boldsymbol{a}}_{0}(0, R)=\hat{\phi}(0, R)=0$. 
Both $\hat{\boldsymbol{a}}_{0}$ and $\hat{\phi}_{1}$ can be determined by solving a differential equation numerically using an iterative process, treating $R$ and $x$ as parameters, respectively. First, the homogeneous form of (29) is solved for each value of $x$ subject to the boundary conditions in (24) and (25). This then provides a first estimate for $\hat{\phi}_{1}$ which is subsequently used in the right-hand sides of (26)-(28). The initial-value problems (26)-(28) are then solved at each value of $r$. A new estimate for $\hat{\phi}_{1}$ is then determined by solving the inhomogeneous form of (29). This process is repeated iteratively until the solutions converge to a specified tolerance.

The full solution is then

$$
\begin{aligned}
\boldsymbol{u}(x, r, \theta, t) & =\left\{\boldsymbol{a}_{0}(x, r) \exp (\mathrm{i} m k(r) x)+\hat{\boldsymbol{a}}_{0, r_{h}}(x, R) E_{h}+\hat{\boldsymbol{a}}_{0, r_{t}}(x, R) E_{t}\right\} \exp (\mathrm{i} m \theta-\mathrm{i} \omega t), \\
\Phi(x, r, \theta, t) & =\frac{1}{m}\left\{\phi_{1}(x, r) \exp (\mathrm{i} m k(r) x)+\hat{\phi}_{1, r_{h}}(x, R) E_{h}+\hat{\phi}_{1, r_{t}}(x, R) E_{t}\right\} \exp (\mathrm{i} m \theta-\mathrm{i} \omega t),
\end{aligned}
$$

where $E_{h, t}=\exp \left(\operatorname{im}\left\{k\left(r_{h, t}\right) x+\left(r-r_{h, t}\right) k_{r}\left(r_{h, t}\right) x\right\}\right)$. The boundary-layer correction terms decay exponentially away from the respective walls, which is to be expected given that they are being driven by a subsonically convecting disturbance in the main body of the flow.

For a potential mean flow with zero mean vorticity, both the general inner solution and boundary-layer correction are simplified. The vorticity part becomes uncoupled from the potential part (see Golubev \& Atassi 2000a) and can be determined analytically. The solution in this case is described in the Appendix.

\subsection{Initial conditions}

For simplicity, the initial wake distribution is described here by a series of Gaussian curves centred on each blade trailing edge, so that the wake distribution is given by

$$
f\left(r, \theta^{\prime}\right)=\sum_{n=0}^{B}-D(r) \exp \left\{-\frac{r^{2}}{2 S^{2}}\left(\theta^{\prime}-\frac{2 n \pi}{B}\right)^{2}\right\},
$$

where $D$ is the depth of the wake and $S$ is the standard deviation of the Gaussian distribution, which determines the width of each blade wake. It is assumed that the initial radial velocity perturbation is zero.

The wake velocity must be resolved into axial and azimuthal components for the initial-value analysis, and the Kutta condition of smooth flow at each trailing edge forces both the steady and unsteady flows to be aligned with the trailing edges. Therefore, if the blades are aligned at an angle $\eta$ to the axial flow at the trailing edge,

$$
\tan \eta=\frac{\left(W-r \Omega_{F}\right)}{U}=\frac{u_{\theta}^{\prime}(0, r)}{u_{x}^{\prime}(0, r)} .
$$

At the blade trailing edge, the gust is assumed to be purely vortical and, after taking the azimuthal Fourier transform of (32), the Fourier coefficients $\beta_{m}(r)$ are assigned (without loss of generality) solely to the vortical part of the disturbance such that

$$
a_{x}(0, r)=\beta_{m}(r) \cos \eta, \quad a_{\theta}(0, r)=\beta_{m}(r) \sin \eta, \quad a_{r}(0, r)=0, \quad \phi(0, r)=0 .
$$

Each harmonic is then propagated downstream according to the evolution equations in $\S 2.1$. 


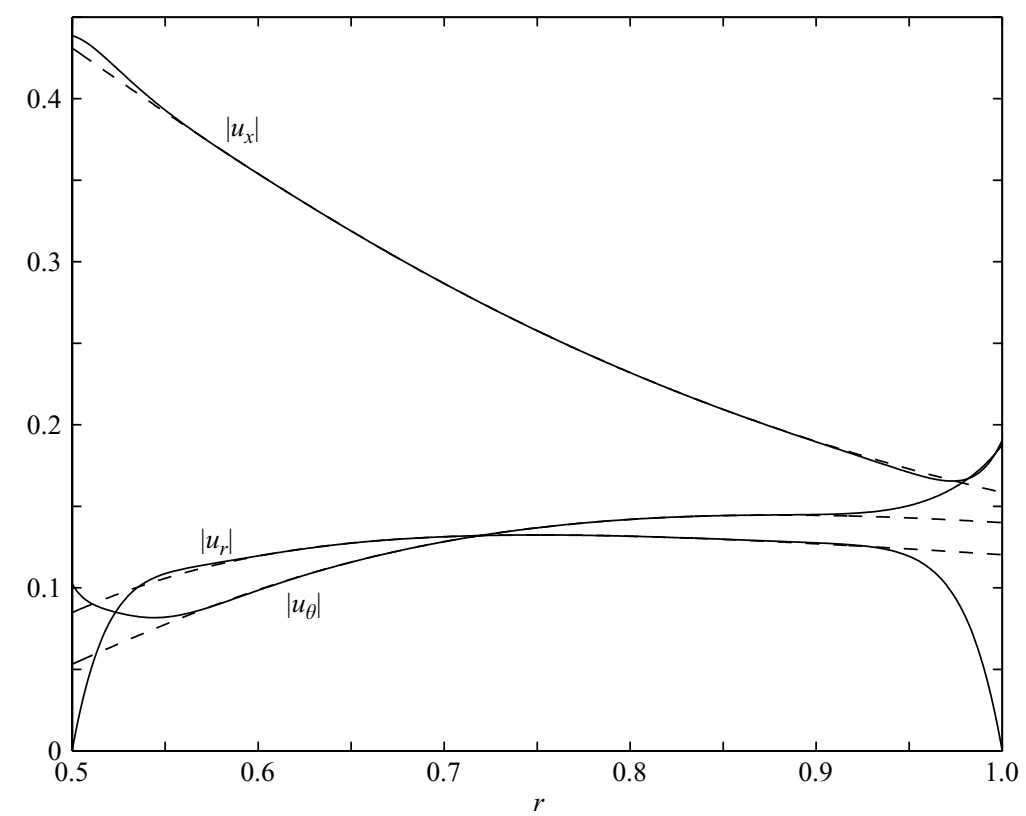

Figure 1. Absolute value of perturbation velocity components for first harmonic $(m=26)$ at $x=0.5$. - - , inner solution only; - , with boundary-layer correction.

\subsection{Example}

As an example, a uniform annulus with hub-tip ratio $r_{h}=0.5$ is taken to represent the duct between the rotor trailing edge and the downstream stator vanes. The mean velocity field is given by

$$
\begin{aligned}
U^{2}(r) & =U_{0}^{2}-2\left[W_{1}^{2}\left(r^{2}-1\right)+2 W_{1} W_{2} \ln (r)\right], \\
W(r) & =W_{1} r+W_{2} / r .
\end{aligned}
$$

where the axial velocity distribution has been obtained by assuming uniform stagnation enthalpy. The values $U_{0}=0.4, W_{1}=0.2$ and $W_{2}=0.1$ are used throughout. The fan is assumed to have $B=26$ blades and rotates with $\Omega_{F}=0.9$. The initial wake is taken to be of uniform depth $(D=1)$ with the width determined by $S=0.01$ (at blade midspan, this value of $S$ is approximately $5 \%$ of the blade pitch). The Fourier coefficients decay exponentially, so that only a finite number of harmonics need be included in the subsequent calculations.

Each of the harmonics produced by the initial wake is propagated downstream as solutions to the initial-value problem given by (20), together with the boundary-layer correction. The effect of this correction is shown in figure 1, which compares solutions for the three perturbation velocity components at $x=0.5$, with and without the boundary-layer solution. The effect of the boundary layer becomes more pronounced the further the disturbance is carried downstream.

At several locations downstream, the wakes are reconstructed, and shown in figure 2. This shows that the wakes become increasingly skewed as they propagate downstream and the cause of this distortion is the development of the local radial wavenumber, which increases linearly with downstream distance as $m x k_{r}$, and the evolution of the amplitudes through (20). The degree of distortion and sense of rotation of the wake depend on the mean flow distribution and fan rotation rate. In this example, $k_{r}(r)$ 

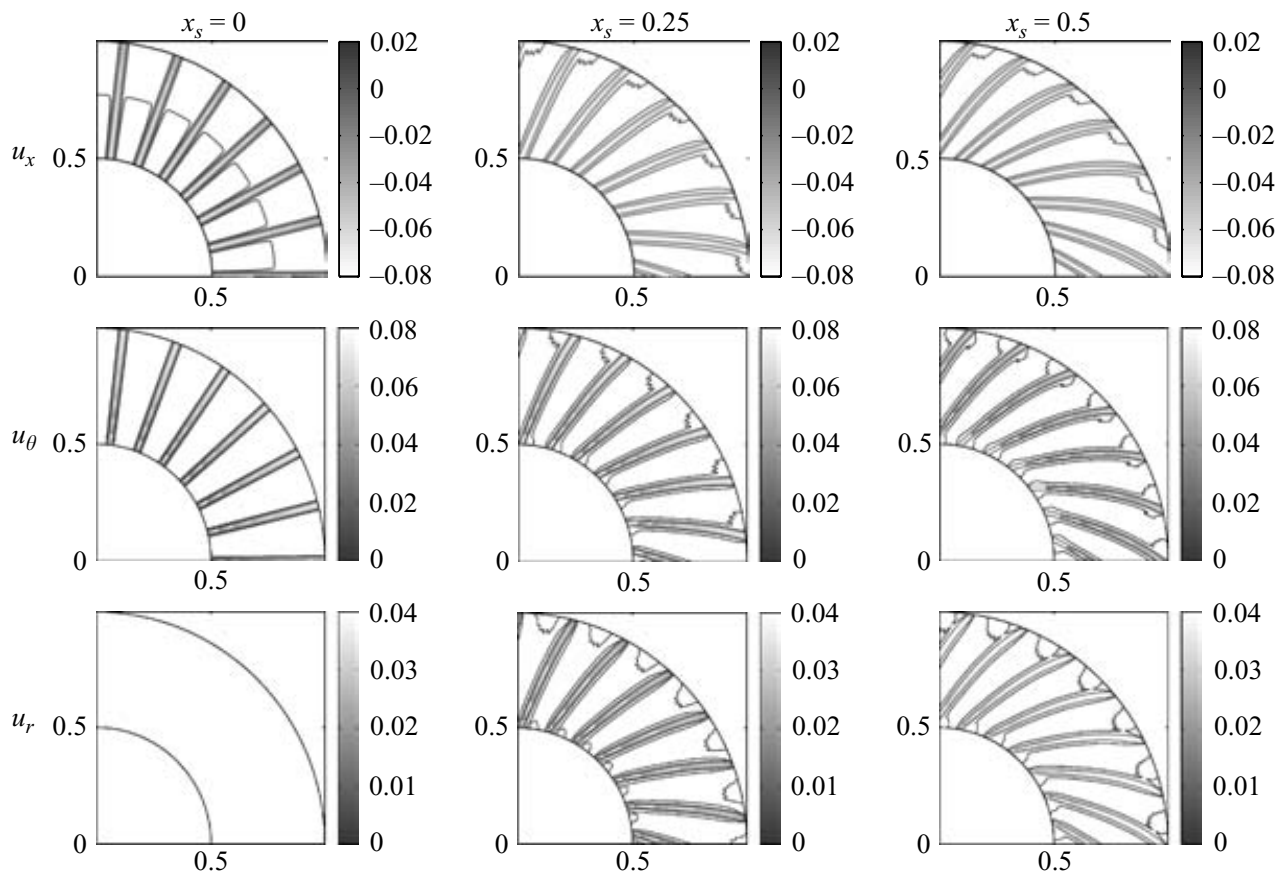

FIGURE 2. Wake reconstruction showing values of the total perturbation velocity components at several locations downstream. The fan is rotating in the anticlockwise direction.

is largest at the hub so that the wake at the hub becomes more distorted from its original position than that at the tip. The skewing of the wakes governs the amount of wake intersection with the downstream stator blades, and this is an important factor in terms of noise generation which will be discussed later. Note also in figure 2 how a significant (mainly outward) radial velocity is generated, even though the wake has zero radial component initially. The effect of the boundary-layer correction near the walls is also apparent, where the wake thickness is significantly increased.

\section{Rotor-wake stator interaction}

Using the theory of $\S 2$, the wake distribution is evolved downstream in the cylindrical duct as far as just upstream of the stator-blade leading edges, located at $x=x_{s}$. The evolved wake is then used as input into a local cascade response scheme in an inner region around the stator blades, and the noise generated locally in the inner region is then matched back onto an upstream-propagating acoustic field in the outer region in the duct. In order to do this, inner and outer coordinates need to be defined and the mean flow calculated, and this is completed in the next subsection.

\subsection{Inner cascade coordinates and mean flow}

Given that the number of stator blades, $V$, is typically larger than the number of blades in the fan, it is consistent to take $V \gg 1$ and introduce a small parameter $\varepsilon=2 \pi / V$ corresponding to the angular separation between adjacent stator blades. Axial and azimuthal inner coordinates, $x^{i}=\left(x-x_{s}\right) / \epsilon, \theta^{i}=\theta / \epsilon$, can now be defined (an inner radial coordinate will also be defined for the unsteady flow, but is not needed here). The mean flow through the stator is composed of two components: one corresponding to the axial and swirling flow generated by the fan upstream; and 
a second corresponding to the distortion generated by the stator-blade geometry. It is supposed that the stator-blade camber, thickness and local angle of attack to the far-upstream flow are small (ordering parameter $\delta$ ), and it therefore follows that the total mean velocity takes the form

$$
U(r) \boldsymbol{e}_{x}+W(r) \boldsymbol{e}_{\theta}+\delta \boldsymbol{u}_{1}\left(r, x^{i}, \theta^{i}\right) .
$$

The third term in (37) corresponds to the effects of the stator row, and is necessarily a function of the inner axial and azimuthal coordinates. The radial dependence of this third term, however, is only through the outer radial coordinate. This follows if it is assumed that the stator-blade geometry changes only on the outer scale, which is entirely reasonable since the leading-edge spacing is linear in $r$, and changes in the blade inclination and/or geometry in response to the radial variation in the incident mean flow will also occur on the outer radial scale. The mean vorticity can now be calculated from (37), and will necessarily be of the form $\xi_{0}+\delta \xi_{1}$, with the stator row providing the $O(\delta)$ correction to the mean vorticity $\xi_{0}$ associated with the incident axial and swirling flow. However, in order for $\delta \xi_{1}$ to remain small, it is easy to show that two restrictions must be placed on $\delta \boldsymbol{u}_{1}$ : first, its radial component must be zero, so that mean radial secondary flows generated by the stator appear only at $O\left(\delta^{2}\right)$; and secondly, that

$$
\frac{1}{r} \frac{\partial \boldsymbol{u}_{1 x}}{\partial \theta^{i}}=\frac{\partial \boldsymbol{u}_{1 \theta}}{\partial x^{i}} .
$$

These two conditions together mean that the steady-flow perturbation induced by the blade row, $\delta \boldsymbol{u}_{1}$, is given to $O(\delta)$ by irrotational thin airfoil theory in the surface of constant $r$.

At a given radius $r=r_{0}$, an inner radial coordinate centred on $r_{0}$ and scaling on $\epsilon$ is defined, so that the inner region itself is of size $O(\epsilon)$ in each of the axial, radial and azimuthal directions. In this inner region, local linear cascade Cartesian coordinates $(X, Y, Z)$ are defined, which are orientated such that the $X$-axis is aligned with the far upstream steady flow (i.e. with the $O(1)$ velocity in (37)), and the $Z$-axis is radial. Specifically,

$$
\begin{aligned}
& X=\left(\frac{r_{t}^{*}}{b^{*}}\right)\left\{\left(x-x_{s}\right) \cos \gamma+r_{0} \theta \sin \gamma\right\} / \varepsilon, \\
& Y=\left(\frac{r_{t}^{*}}{b^{*}}\right)\left\{r_{0} \theta \cos \gamma-\left(x-x_{s}\right) \sin \gamma\right\} / \varepsilon, \\
& Z=\left(\frac{r_{t}^{*}}{b^{*}}\right)\left\{r-r_{0}\right\} / \varepsilon,
\end{aligned}
$$

where the local blade semi-chord $b^{*}=b^{*}\left(r_{0}\right)$ is used for non-dimensionalization and $\tan \gamma=W^{*} / U^{*}$. The steady-flow speed far upstream in the inner region, $U_{\infty}^{*}\left(r_{0}\right)$, is then related to the duct mean flow speed by

$$
U_{\infty}^{*}\left(r_{0}\right)=\left[U^{*}\left(r_{0}\right)^{2}+W^{*}\left(r_{0}\right)^{2}\right]^{1 / 2} .
$$

Note that $U_{\infty}^{*}\left(r_{0}\right)$ depends on the outer radial coordinate, and note also that the mean-flow distortion due to the blade row, $\delta \boldsymbol{u}_{1}$ decays upstream in the inner region, so that in the local Cartesian coordinates the steady velocity approaches $\left(U_{\infty}^{*}, 0,0\right)$ as $X \rightarrow-\infty$. The corresponding Mach number is $M_{\infty}=U_{\infty}^{*} / c_{\infty}^{*}$, where $c_{\infty}^{*}$ is the local sound speed. In the Cartesian $(X, Y, Z)$ coordinates the leading-order mean 


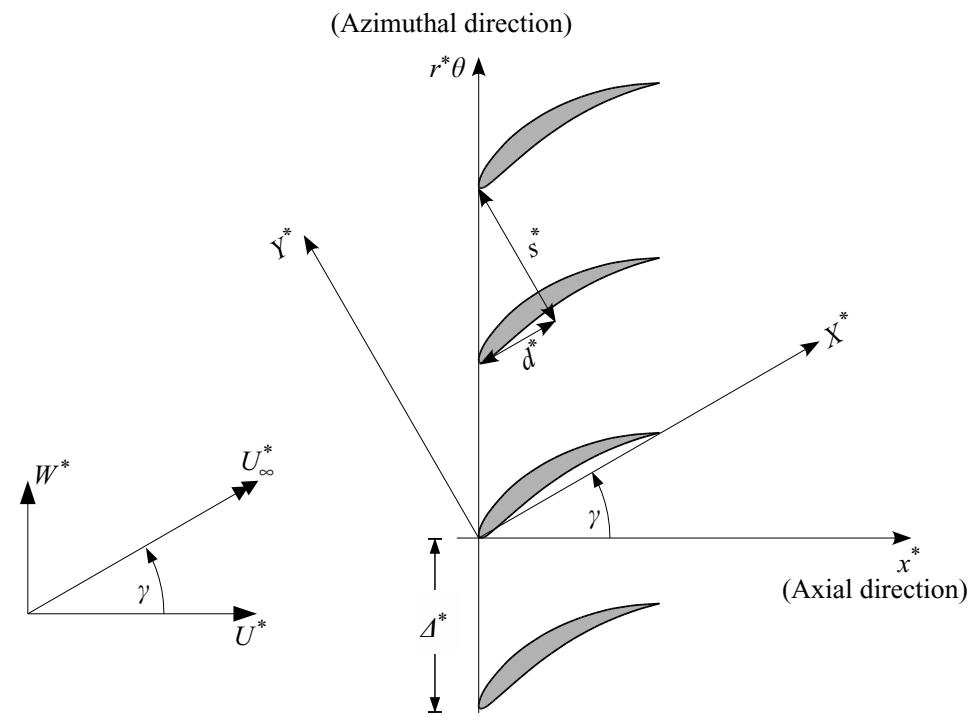

FIGURE 3. Local cascade coordinate system $\left(X^{*}, Y^{*}\right)$, with cascade stagger angle given by $\alpha^{*}=\tan ^{-1}\left(s^{*} / d^{*}\right)$.

vorticity is

$$
\boldsymbol{\xi}_{0}=\left(0,-\frac{1}{U_{\infty}^{*}} \frac{\partial U_{\infty}^{*}}{\partial Z}, 0\right) .
$$

Figure 3 shows the staggered cascade in physical space and its relation to the duct geometry.

It has already been noted that the $O(\delta)$ steady disturbance flow generated by the blade row is irrotational in surfaces of constant $r$, and has zero radial component. The asymptotic cascade model of Evers \& Peake (2002) for non-swirling mean flow accounts for the blade geometry using thin-airfoil theory $(\delta \ll 1)$, and it therefore follows that their mean flow can be used in the current problem as well. Specifically, in the inner region, the physical $(X, Y, Z)$ coordinates are transformed to steady potential-streamfunction coordinates $(\phi, \psi, Z)$, with

$$
\phi=\frac{X^{*}}{b^{*}}+\delta \operatorname{Re}[F]+O\left(\delta^{2}\right), \quad \psi=\beta_{\infty} \frac{Y^{*}}{b^{*}}+\delta \operatorname{Im}[F]+O\left(\delta^{2}\right),
$$

where $\delta b^{*} U_{\infty}^{*} F(X, Y)$ is the complex disturbance potential describing the twodimensional flow through the cascade at a given radius. This steady disturbance flow is given by $F=F_{i} / \beta_{\infty}$, where $F_{i}$ is the equivalent incompressible complex potential and $\beta_{\infty}=\sqrt{1-M_{\infty}^{2}}$. Hence, the mean flow disturbance, $\delta \boldsymbol{u}_{1}$, induced by the presence of the stator blades is calculated using thin-airfoil theory at each radius, with radial variations appearing through the parametric dependence of $U_{\infty}^{*}, b^{*}$ and the cascade geometry on the outer radial coordinate $r_{0}$.

\subsection{Unsteady flow far upstream in the inner region}

In $\S 2$, it was shown how the Fourier components of rotor wakes are distorted by the mean swirl in the outer duct flow. The aim now is to consider how these components, referred to here as gusts, are distorted as they propagate through the inner region toward the stator blades. In the inner region, the total unsteady velocity is written

$$
U_{\infty}^{*}\left(A_{t}, A_{n}, A_{z}\right) \exp \left(\mathrm{i} K\left[X+k_{n} \beta_{\infty} Y+k_{3} Z-t\right]\right) \quad \text { as } \quad X \rightarrow-\infty,
$$


where $K=\omega^{*} b^{*} / U_{\infty}^{*}$ is the aerodynamic reduced frequency based on the total flow speed onto the local blade. Here, the time $t$ has been made non-dimensional using the local time scale $b^{*} / U_{\infty}^{*}$. Using matched asymptotic expansions, (45) must match the inner limit of the outer unsteady velocity, which is given by

$$
c_{0}^{*}\left(B_{x}, B_{\theta}, B_{r}\right)\left(x, r_{0}\right) \exp (\mathrm{i}[m k(r) x+m \theta-\omega t]),
$$

as $x \rightarrow x_{s}, r \rightarrow r_{0}$. Here, to leading order in $m$, the vector $\boldsymbol{B}$ is given as a sum of contributions from the outer unsteady vortical velocity, the outer potential unsteady velocity and the boundary-layer correction described in $\S 2.2$ (i.e. $\boldsymbol{B}$ follows from $\boldsymbol{u}+\nabla \Phi$, with $\boldsymbol{u}$ and $\Phi$ given by (30) and (31)). Matching the amplitudes in (45) and (46) gives

$$
A_{t}=Q\left(r_{0}\right)\left(B_{x} \cos \gamma+B_{\theta} \sin \gamma\right), \quad A_{n}=Q\left(r_{0}\right)\left(B_{\theta} \cos \gamma-B_{x} \sin \gamma\right), \quad A_{z}=Q\left(r_{0}\right) B_{r},
$$

where

$$
Q\left(r_{0}\right)=\frac{1}{M_{\infty} c_{0}} \exp \left(\mathrm{imk}\left(r_{0}\right) x_{s}\right), \quad \tan \gamma=W / U,
$$

and $c_{0}=c_{\infty}^{*} / c_{0}^{*}$. Matching the phases in (45) and (46) determines the cascade wavenumbers

$$
K=\frac{\omega}{M_{\infty} c_{0}}\left(\frac{b^{*}}{r_{t}^{*}}\right), \quad \beta_{\infty} k_{n}=\frac{m}{K r \cos \gamma}\left(\frac{b^{*}}{r_{t}^{*}}\right)-\tan \gamma, \quad k_{3}=\frac{k_{r}}{K}\left(\frac{b^{*}}{r_{t}^{*}}\right) .
$$

It is important to note that (45) describes a three-dimensional gust, which accounts for the fast variation along the blade radius through the phase term $k_{3} Z$, and for the local variation through the dependence of the various parameters $\left(b^{*}, M_{\infty}, \boldsymbol{B}, \ldots\right)$ on $r_{0}$.

In the inner region, the blade interaction problem has been reduced to an equivalent gust-cascade problem. The input into this cascade problem is in the form of the gust (45) at upstream infinity, in which the amplitude vector $\boldsymbol{A}$ and the radial wavenumber $k_{3}$ have been determined by matching with the outer duct flow upstream. The cascade problem will be completed using asymptotic analysis in the limit of high local reduced frequency, $K \gg 1$, and since $\omega=m \Omega_{F}$, the assumptions of large $K$ and $m$ are equivalent. (Note that application of large $K$ means that the cascade solidity is large.) The preferred limit $K \delta=O(1)$ is assumed.

\subsection{Distortion of incident unsteady flow in the inner region}

This section deals with how the wake field incident from upstream is distorted as it propagates in the inner region. The unsteady velocity field corresponding to the disturbance imposed from upstream is split into vortical and potential parts, as in $\S 2$, with

$$
\begin{aligned}
\boldsymbol{u} & =\left(\boldsymbol{A}_{0}+\delta \boldsymbol{A}_{1}\right) \exp \left(\mathrm{i} K\left(p_{0}+\delta p_{1}\right)-\mathrm{i} K t\right), \\
\Phi & =\frac{\delta}{K} \Phi_{1} \exp \left(\mathrm{i} K\left(p_{0}+\delta p_{1}\right)-\mathrm{i} K t\right) .
\end{aligned}
$$

Here, $\boldsymbol{A}_{0}$ corresponds exactly to the amplitude vector given in (47), and is therefore read straight from the solution of the outer problem, i.e. the field which would have been present in the absence of the stator blades. The distorting effect of the stator blades appears in the $O(\delta)$ correction to the amplitude, $\delta \boldsymbol{A}_{1}$. Since the potential field associated with the wake disturbance in the outer region has been absorbed into the term $\boldsymbol{A}_{0}$, the additional potential induced by the interaction between the mean 
vortical flow and $\delta \boldsymbol{A}_{1}$ must be of size $O(\delta / K)$; the $\delta$ factor corresponding to size of the mean-flow distortion and the $1 / K$ corresponding to the scaling expected for the potential of a nearly convected disturbance (cf. equations (11) and (13) in the outer region). In (50) and (51), the phase of the disturbances comprises a leading-order term $K p_{0}$, corresponding to propagation through uniform flow, and the correction term $K \delta p_{1}$ arising from the distorting effect of the cascade non-uniform mean flow.

The unsteady flow in the inner region is governed by the coupled vorticity and wave equations (6) and (7) written in terms of the inner $(X, Y, Z)$ coordinates. However, rather than solving these equations in $(X, Y, Z)$, we will follow Myers \& Kerschen (1995) and work throughout in the steady cascade potential-streamfunction coordinates $\phi, \psi$ together with the spanwise coordinate $Z$. Substitution of the velocity field (50) and (51) into the vorticity equation (6), and then considering just the $O(K)$ terms gives the equation

$$
-1+(1+\delta q)^{2} \frac{\partial}{\partial \phi}\left(p_{0}+\delta p_{1}\right)=0
$$

where $U_{\infty}^{*} \delta q$ is the perturbation to the uniform mean flow induced by the cascade. Equation (52) is solved for the phase in the form

$$
p_{0}+\delta p_{1}=\phi+k_{n} \psi+k_{3} Z+\delta g(\phi, \psi)-\delta\left(\operatorname{Re}[F(-\infty)]+k_{n} \operatorname{Im}[F(-\infty)]\right),
$$

where the term $k_{n} \psi+k_{3} Z$ and the terms involving $F(-\infty)$ have arisen as constants of integration, and have been chosen so as to match with the far-upstream form of the incident velocity (45). Furthermore,

$$
g(\phi, \psi)=-\int_{-\infty}^{\phi} 2 q\left(\phi^{\prime}, \psi\right) \mathrm{d} \phi^{\prime}
$$

is Lighthill's drift function. This result for the phase compares exactly with the result of Myers \& Kerschen $(1995$, equations $(2.5 b, c))$ in the absence of mean vorticity.

The unknown amplitude vectors in (50) are now determined. Introducing the notation $\boldsymbol{A}_{0}=\left(A_{t}^{(0)}, A_{n}^{(0)}, A_{z}^{(0)}\right)$ and $\boldsymbol{A}_{1}=\left(A_{t}^{(1)}, A_{n}^{(1)}, A_{z}^{(1)}\right)$ for the components in the $\phi$, $\psi$ and $Z$-directions, and substituting (51) into the coupled wave equation (7) yields at $O(K)$ the amplitude of the potential field

$$
\Phi_{1}=\frac{\mathrm{i}}{\lambda^{2}}\left[A_{t}^{(1)}+\beta_{\infty} k_{n} A_{n}^{(1)}+k_{3} A_{z}^{(1)}\right]+\frac{\mathrm{i}}{\lambda^{2}}\left[-q A_{t}^{(0)}+\beta_{\infty} \frac{\partial g}{\partial \psi} A_{n}^{(0)}+\beta_{\infty}^{3} q k_{n} A_{n}^{(0)}\right],
$$

where $\lambda^{2}=1+\beta_{\infty}^{2} k_{n}^{2}+k_{3}^{2}$. This potential field corresponds to a hydrodynamic field which is generated by the volume-source term on the right-hand side of (7), with phase which convects with the local mean flow (see also Myers \& Kerschen 1995, equation (3.28)). The equations governing $\delta \boldsymbol{A}_{1}$ are obtained by substituting (50) and (55) into the coupled vorticity equation (6), and taking $O(1)$ terms to yield the transport equation

$$
\begin{aligned}
& \frac{\partial A_{t}^{(1)}}{\partial \phi}=-A_{t}^{(0)} \frac{\partial q}{\partial \phi}-\zeta k_{3} A_{t}^{(1)}-\zeta k_{3}^{2} A_{z}^{(1)}-\zeta k_{3} \eta, \\
& \frac{\partial A_{n}^{(1)}}{\partial \phi}=A_{n}^{(0)} \beta_{\infty}^{2} \frac{\partial q}{\partial \phi}-2 \beta_{\infty} A_{t}^{(0)} \frac{\partial q}{\partial \psi}, \\
& \frac{\partial A_{z}^{(1)}}{\partial \phi}=\zeta A_{t}^{(1)}+\zeta k_{3} A_{z}^{(1)}+\zeta \eta,
\end{aligned}
$$


where

$$
\zeta=\frac{1}{\lambda^{2} U_{\infty}^{*}} \frac{\partial U_{\infty}^{*}}{\partial Z}, \quad \eta(\phi, \psi)=\beta_{\infty} k_{n} A_{n}^{(1)}-q A_{t}^{(0)}+\beta_{\infty} \frac{\partial g}{\partial \psi} A_{n}^{(0)}+\beta_{\infty}^{3} k_{n} q A_{n}^{(0)} .
$$

The solution to (56)-(58) is obtained by integrating (57) directly to determine $A_{n}^{(1)}$. This is then used to simplify (56) and (57). The result is that

$$
\begin{aligned}
& A_{t}^{(1)}=-A_{t}^{(0)} q-\zeta k_{3} \int_{-\infty}^{\phi} \eta\left(\phi^{\prime}\right) \mathrm{d} \phi^{\prime}+\zeta k_{3} A_{t}^{(0)} \int_{-\infty}^{\phi} q\left(\phi^{\prime}\right) \mathrm{d} \phi^{\prime}, \\
& A_{n}^{(1)}=\beta_{\infty} A_{t}^{(0)} \frac{\partial g}{\partial \psi}+A_{n}^{(0)} \beta_{\infty}^{2} q, \\
& A_{z}^{(1)}=\zeta \int_{-\infty}^{\phi} \eta\left(\phi^{\prime}\right) \mathrm{d} \phi^{\prime}-\zeta A_{t}^{(0)} \int_{-\infty}^{\phi} q\left(\phi^{\prime}\right) \mathrm{d} \phi^{\prime} .
\end{aligned}
$$

At this point, it is worth pausing to consider the effect the mean vorticity has had on the results. If $\zeta=0$, then it is easy to see that the expressions for the amplitude correction $\delta \boldsymbol{A}_{1}$ are significantly simplified, and in fact agree exactly with what has been derived previously by Myers \& Kerschen (1995) in irrotational mean flow. The effect of $\zeta \neq 0$ is to introduce the coupling term between the vortical and potential unsteady flows on the right-hand side of (6), and in the analysis this manifests itself as the term $\Phi_{1}$ producing the terms proportional to $\zeta$ on the right-hand sides of (56)-(58). Physically, this means that the $O(\delta)$ potential field carried along by the nearly convected gust stretches the local mean vorticity so as to produce an extra component of unsteady vortical velocity.

\subsection{Gust-leading-edge interaction}

The upstream acoustic field produced when the distorted gust described in $\S 3.3$ interacts with just one of the airfoils is now determined. In the presence of mean vorticity, the upstream-radiated velocity field from the airfoil consists of pressuredominated acoustic-vorticity waves, and is expressed in terms of a potential part $G$ and a vortical part $\boldsymbol{C}$ as

$$
\boldsymbol{u}^{\prime}=\nabla G+\boldsymbol{C}
$$

A modified unsteady velocity potential, $h(\phi, \psi)$, and a modified vortical part of the unsteady velocity, $\boldsymbol{c}(\phi, \psi)$, are introduced, such that

$$
[G, C]=[h(\phi, \psi), \boldsymbol{c}(\phi, \psi)] \exp \left(\mathrm{i} K\left[k_{3} Z-t-M_{\infty}^{2} \phi / \beta_{\infty}^{2}\right]\right) \exp \left(\delta M_{\infty}^{2} q\right) .
$$

Myers \& Kerschen (1995) and Peake \& Kerschen (1997) consider Goldstein's wave equation, (7), in the limit of small $\delta$. In the presence of mean vorticity, this equation becomes

$$
L_{0}(h)+\delta L_{1}(h)=\delta K S(\phi, \psi) \mathrm{e}^{\mathrm{i} K \Omega}+K T_{1}(\boldsymbol{c})+K \delta T_{2}(\boldsymbol{c})
$$

where $L_{0}, L_{1}$ and $\Omega$ are the same as in equations $(2.4 c),(2.4 d)$ and $(2.5 b)$ of Myers \& Kerschen (1995). The effects of the mean vorticity lead to modification of the source term, $S(\phi, \psi)$, which is associated with the interaction between the incoming incident gust and the non-uniform mean flow. Specifically,

$$
\begin{aligned}
S(\phi, \psi)= & \frac{2}{\beta_{\infty}^{2}}\left\{\mathrm{i}\left(A_{t}^{(0)}-k_{n} A_{n}^{(0)} \beta_{\infty}^{3}\right) q+\mathrm{i}\left(k_{n} A_{t}^{(0)} \beta_{\infty}^{2}+A_{n}^{(0)} \beta_{\infty}\right) \mu\right. \\
& \left.+\frac{1}{K}\left[M_{\infty}^{2} A_{t}^{(0)} \frac{\partial q}{\partial \phi}+M_{\infty}^{2} \beta_{\infty} A_{n}^{(0)} \frac{\partial q}{\partial \psi}+\frac{\zeta k_{3}}{2}\left(\eta-A_{t}^{(0)} q\right)\right]\right\},
\end{aligned}
$$


where the quantity $\beta_{\infty} \delta \mu$ is the perturbation flow angle in the physical plane. In (66), the term involving $\zeta$ corresponds to the effect of mean vorticity (when $\zeta=0$, equation (66) reduces exactly to (2.5a) of Myers \& Kerschen (1995) for irrotational mean flow). Furthermore, the presence of mean vorticity leads to the second and third source terms on the right-hand side of (65), corresponding to the interaction between the vortical part of the outgoing acoustic field and the non-uniform mean flow. These source terms are given by

$$
\begin{aligned}
& T_{1}(\boldsymbol{c})=-\frac{1}{\beta_{\infty}^{2}}\left\{-\frac{\mathrm{i} M_{\infty}^{2}}{\beta_{\infty}^{2}} c_{t}+\mathrm{i} k_{3} c_{z}+\frac{1}{K}\left[\frac{\partial c_{t}}{\partial \phi}+\beta_{\infty} \frac{\partial c_{n}}{\partial \psi}\right]\right\}, \\
& T_{2}(\boldsymbol{c})=-\frac{1}{\beta_{\infty}^{2}}\left\{-\frac{\mathrm{i} M_{\infty}^{2}}{\beta_{\infty}^{2}} q c_{t}+\frac{1}{K}\left[-\beta_{\infty}^{2} \frac{\partial q}{\partial \phi} c_{t}+\beta_{\infty}^{3} q \frac{\partial c_{n}}{\partial \psi}-\beta_{\infty} \frac{\partial q}{\partial \psi} c_{n}\right]\right\} .
\end{aligned}
$$

With the unsteady velocity decomposition (63), the zero normal velocity boundary condition on the blade surface, $\psi=0$, becomes

$$
\frac{\partial h}{\partial \psi}+c_{n}+\delta M_{\infty}^{2} \frac{\partial q}{\partial \psi} h=-\left[\frac{A_{n}^{(0)}}{\beta_{\infty}}\left(1-\delta M_{\infty}^{2} q\right)-\delta 2 A_{t}^{(0)} \mu\right] \mathrm{e}^{\mathrm{i} K \Omega},
$$

which is to be compared to (2.6) of Myers \& Kerschen (1995). The additional feature here is the term $c_{n}$, corresponding to the blade upwash of the vortical part of the velocity associated with the upstream-propagating acoustic wave.

Following Myers \& Kerschen (1995), a local leading-edge region, scaling on the gust wavelength, around the blade leading edge is now considered. In this local leadingedge region, the leading-edge coordinates $(\Phi, \Psi) \equiv k(\phi, \psi)$ are introduced, and the wave equation (65) is transformed to

$$
K^{2}\left\{L_{0}(h)+\delta K^{1 / 2} L_{1}(h)\right\}=\delta K^{3 / 2} \hat{S}(\Phi, \Psi) \mathrm{e}^{\mathrm{i} K \Omega}+K \hat{T}_{1}(\boldsymbol{c})+\delta K^{3 / 2} \hat{T}_{2}(\boldsymbol{c}),
$$

where the circumflex indicates that $(1 / K) \partial / \partial \phi$ terms have become $\partial / \partial \Phi$, etc. The fractional power $K^{3 / 2}$ multiplying $\hat{S}$ has arisen because in the blade leading-edge region, $q$ has a square-root singularity, so that the mean-flow perturbation speed, $\delta q$, is $O\left(\delta K^{1 / 2}\right)$. In the analysis of Myers \& Kerschen (1995) the modified unsteady potential in the leading-edge region is expanded as

$$
h=\frac{1}{K}\left[H_{0}+\delta K^{1 / 2}\left(H_{1}+H_{2}+H_{3}\right)+O\left(\delta^{2} K, \delta\right)\right],
$$

where the terms $\mathrm{H}_{0-3}$ account for different physical effects near the leading edge, as described in Myers \& Kerschen $(1995,1997)$ and Tsai \& Kerschen (1990). In the presence of mean vorticity, the additional expansion of the unsteady vortical velocity associated with $h$ must be introduced, namely

$$
\boldsymbol{c}=\frac{1}{K}\left[\boldsymbol{c}_{0}+\delta K^{1 / 2} \boldsymbol{c}_{1}+O\left(\delta^{2} K, \delta\right)\right]
$$

This choice for the scaling of $c$ ensures that the upstream-radiation is pressuredominated. Substitution into (70) yields the equation

$$
\begin{aligned}
L_{0}\left(H_{0}\right)+\delta K^{1 / 2}\left\{L _ { 0 } \left(H_{1}\right.\right. & \left.\left.+H_{2}+H_{3}\right)+L_{1}\left(H_{0}\right)\right\}=\delta K^{1 / 2} \hat{S}(\Phi, \Psi) \mathrm{e}^{\mathrm{i} K \Omega} \\
& +\frac{1}{K} \hat{T}_{1}\left(\boldsymbol{c}_{0}+\delta K^{1 / 2} \boldsymbol{c}_{1}\right)+\delta K^{-1 / 2} \hat{T}_{2}\left(\boldsymbol{c}_{0}+\delta K^{1 / 2} \boldsymbol{c}_{1}\right),
\end{aligned}
$$


and the boundary condition (69) transforms to

$$
\begin{aligned}
\frac{\partial H_{0}}{\partial \Psi}+\delta K^{1 / 2} \frac{\partial}{\partial \Psi}\left\{H_{1}+H_{2}+H_{3}\right\} & +\delta M_{\infty}^{2} \frac{\partial q}{\partial \Psi} H_{0}+\frac{c_{n}}{K} \\
& =-\left[\frac{A_{n}^{(0)}}{\beta_{\infty}}\left(1-\delta M_{\infty}^{2} q\right)-\delta 2 A_{t}^{(0)} \mu\right] \mathrm{e}^{\mathrm{i} K \Omega} .
\end{aligned}
$$

In our asymptotic analysis of the noise generation, and following the approach of Myers \& Kerschen (1995), perturbation terms of size $O(1)$ and $O\left(\delta K^{1 / 2}\right)$ in (73) and (74) are retained, while terms of size $O\left(K^{-1}\right), O(\delta), O\left(\delta^{2} K\right)$ and $O\left(\delta K^{-1 / 2}\right)$ are neglected. Therefore, all the terms involving the vortical velocity associated with the outgoing acoustic field, $\boldsymbol{c}$, are neglected in (73) and (74), so that $h$ becomes decoupled from $\boldsymbol{c}$. In addition, the contribution to $\hat{S}(\Phi, \Psi)$ due to the presence of mean vorticity, i.e. the term in (66) which is proportional to $\zeta$, is also neglected. This point follows because the $\zeta$-independent terms in (66) are $O\left(K^{1 / 2}\right)$ in the leading-edge region (since $q, \mu=O\left(K^{1 / 2}\right)$ there). In contrast, the $\zeta$-dependent term in (66) is $O\left(K^{-1 / 2}\right)$. Hence, the $\zeta$-dependent source term appears as a term of size $O\left(\delta K^{-1 / 2}\right)$ on the right-hand side of (73), and is therefore neglected.

It can now be seen that the leading-edge noise-generation problem is identical to the sound-generation problem studied in Myers \& Kerschen $(1995,1997)$ and Tsai \& Kerschen (1990), since the effects of the presence of the mean vorticity have been seen to be confined to terms which are neglected to the asymptotic order considered. The only point still to be checked is the effect of the mean vorticity on the acoustic waves as they propagate away from the airfoil. A leading-edge ray solution in the form

$$
\begin{aligned}
& h_{l}=K^{-3 / 2} A_{l}(r, \theta) \mathrm{e}^{\mathrm{i} K \sigma(r, \theta)}+O\left(K^{-5 / 2}\right), \\
& \boldsymbol{a}_{l}=K^{-3 / 2} \boldsymbol{b}_{l}(r, \theta) \mathrm{e}^{\mathrm{i} K \sigma(r, \theta)}+O\left(K^{-5 / 2}\right),
\end{aligned}
$$

is sought, where $(r, \theta)$ are the polar form of the coordinates $(\phi, \psi)$. The expression for the modified unsteady velocity potential $h_{l}$ is exactly the leading-edge ray field used by Myers \& Kerschen (1995, equation (3.30)), while the expression for $\boldsymbol{a}_{l}$ is the corresponding vortical part of the unsteady velocity. In principle, $\boldsymbol{a}_{l}$ is coupled to $h_{l}$ via the presence of mean vorticity. However, substituting (75) and (76) into the wave equation (7) leads to the same eikonal equation for the phase $\sigma$ as found by Myers \& Kerschen (1995, equation (3.31)). However, the transport equation for the amplitudes is modified, and becomes

$$
\begin{aligned}
\frac{\partial \sigma}{\partial r} & \frac{\partial A_{l}}{\partial r}+\frac{1}{r} \frac{\partial \sigma}{\partial \theta} \frac{\partial A_{l}}{\partial \theta}+\frac{A_{l}}{2}\left[\frac{1}{r} \frac{\partial}{\partial r}\left(r \frac{\partial \sigma}{\partial r}\right)+\frac{1}{r^{2}} \frac{\partial^{2} \sigma}{\partial \theta^{2}}\right]+O(\delta) \\
= & -\frac{1}{2 \beta_{\infty}^{2}}\left[\left(-\frac{M_{\infty}^{2}}{\beta_{\infty}^{2}}+\sin \theta \frac{\partial \sigma}{\partial r}+\frac{\cos \theta}{r} \frac{\partial \sigma}{\partial \theta}\right) b_{\phi}+k_{3} b_{z}\right. \\
& \left.+\beta_{\infty}\left(\cos \theta \frac{\partial \sigma}{\partial r}-\frac{\sin \theta}{r} \frac{\partial \sigma}{\partial \theta}\right) b_{\psi}\right]+O(\delta) .
\end{aligned}
$$

This is to be compared with equation (3.32) of Myers \& Kerschen (1995), in which the vortical terms involving $\boldsymbol{b}_{l}$ are absent. Substituting (75) and (76) into the vorticity equation (6) and taking just the leading-order terms yields

$$
\begin{aligned}
& \left(-1+\sin \theta \frac{\partial \sigma}{\partial r}-\frac{M_{\infty}^{2}}{\beta_{\infty}^{2}}\right) b_{\phi}=\frac{1}{U_{\infty}^{*}} \frac{\partial U_{\infty}^{*}}{\partial Z} k_{3} A_{l}, \\
& \left(-1+\sin \theta \frac{\partial \sigma}{\partial r}-\frac{M_{\infty}^{2}}{\beta_{\infty}^{2}}\right) b_{\psi}=0,
\end{aligned}
$$




$$
\left(-1+\sin \theta \frac{\partial \sigma}{\partial r}-\frac{M_{\infty}^{2}}{\beta_{\infty}^{2}}\right) b_{z}=-\frac{1}{U_{\infty}^{*}} \frac{\partial U_{\infty}^{*}}{\partial Z}\left(-\frac{M_{\infty}^{2}}{\beta_{\infty}^{2}}+\sin \theta \frac{\partial \sigma}{\partial r}\right) A_{l} .
$$

From the eikonal equation, it follows that $\partial \sigma / \partial \theta=O(\delta)$ (see Myers \& Kerschen 1995, equation (3.34)), and substituting for $b_{\phi}, b_{\psi}$ and $b_{z}$ from (78)-(80) into (77) then reduces the right-hand side of (77) to zero, and recovers the transport equation (3.32) of Myers \& Kerschen (1995). Thus, the outer leading-edge acoustic potential becomes decoupled from the vortical part of the velocity field, so that, to the asymptotic order considered, the local mean vorticity has no effect on the propagation of acoustic waves away from the leading edge.

In summary, the effects of the mean vorticity do not appear at the relative orders considered in the asymptotic analysis; neither in the sound generation in the blade leading-edge region, nor in the propagation of acoustic waves away from the leading edge within the inner region. Therefore, the results obtained by Peake $\&$ Kerschen (1997) and Evers \& Peake (2002) for the gust-cascade interaction in the absence of swirl can be applied here, and this will be described in the next subsection.

\subsection{Radiation produced by the cascade interaction}

So far the acoustic response of a single blade has been calculated. In the case of zero swirl, Peake \& Kerschen (1997) and Evers \& Peake (2002) have shown how the single-blade response can be used to construct the total radiated field upstream of the cascade. Since it has been shown, at the end of the last subsection, that the presence of mean vorticity does not affect the propagation of acoustic waves in the inner region upstream of the cascade to the asymptotic order considered, the zero-swirl results can be used here. The acoustic potential can be expressed in terms of a superposition of radiating plane-wave modes in the form

$h(\phi, \psi) \sim Q(r) \exp \left(-\mathrm{i} K \delta\left(\operatorname{Re}[F(-\infty)]+k_{n} \operatorname{Im}[F(-\infty)]\right)\right) \sum_{n=n_{r}}^{n_{q}} R_{n}(r) \exp \left(-\mathrm{i} \sigma_{n} \phi-\mathrm{i} \eta_{n} \psi\right)$,

where

$$
\begin{aligned}
& \sigma_{n}=\left\{\left(2 n \pi-\sigma^{\prime}\right) \sin \alpha+\cos \alpha\left[(\Delta K w)^{2}-\left(2 n \pi-\sigma^{\prime}\right)^{2}\right]^{1 / 2}\right\} / \Delta, \\
& \eta_{n}=\left\{\left(2 n \pi-\sigma^{\prime}\right) \cos \alpha-\sin \alpha\left[(\Delta K w)^{2}-\left(2 n \pi-\sigma^{\prime}\right)^{2}\right]^{1 / 2}\right\} / \Delta,
\end{aligned}
$$

with

$$
\alpha=\tan ^{-1}\left(\beta_{\infty} \tan \alpha^{*}\right), \quad \Delta=\left(d^{* 2}+\beta_{\infty}^{2} s^{* 2}\right)^{1 / 2} / b^{*},
$$

and

$$
\sigma^{\prime}=\frac{K\left(d^{*}+\beta_{\infty} k_{n} s^{*}\right)}{b^{*}}+\frac{K M_{\infty}^{2} d^{*}}{b^{*} \beta_{\infty}^{2}} .
$$

The quantity $K w$ is the acoustic reduced frequency with

$$
w^{2}=\left(M_{\infty} / \beta_{\infty}^{2}\right)^{2}-\left(k_{3} / \beta_{\infty}\right)^{2} .
$$

Expressions for the modal coefficients $R_{n}$ and details of the steady-flow calculation can be found in Evers \& Peake (2002). The values $n_{r}$ and $n_{q}$ are the lowest and highest values of $n$ such that the square root in (82) and (83) is real. Each element in the sum then corresponds to a forward-radiated plane-wave mode propagating in a different direction away from the cascade. The case where the quantity in the square root is zero corresponds precisely to waves propagating along the front face of the cascade. 
The dimensional acoustic pressure field, $p_{c}^{*}$, associated with the sound radiation upstream of the cascade is defined in Peake \& Kerschen (1997) as

$$
p_{c}^{*}=-\rho_{\infty}^{*} U_{\infty}^{*}\left[\frac{\partial h}{\partial \phi}-\mathrm{i} \frac{K}{\beta_{\infty}^{2}} h\right] \exp \left[\left(\mathrm{i} K\left[k_{3} Z-t-M_{\infty}^{2} \phi / \beta_{\infty}^{2}\right]\right),\right.
$$

so that the far-upstream limit of the acoustic pressure in the inner region can easily be recovered from (81).

\subsection{Radiation upstream of the stator row in the outer region}

The final step in the calculation is to match the radiation propagating ahead of the cascade in the inner region, given in (81), onto an upstream-propagating sound field in the cylindrical duct. The unsteady pressure field in the duct can be written as a modal expansion in the form

$$
p^{*}(x, r, \theta, t)=\rho_{0}^{*} c_{0}^{* 2} \sum_{\hat{m}=-\infty}^{\infty} \sum_{s=1}^{\infty} \hat{p}_{\hat{m} s} \mathscr{L}_{\hat{m} s}(r) \exp \left\{\mathrm{i}\left[k_{\hat{m} s}^{-} x+\hat{m} \theta-\omega t\right]\right\},
$$

where $k_{\hat{m} s}^{-}$and $\mathscr{L}_{\hat{m} s}$ are the eigenvalues and eigenfunctions, respectively, corresponding to nearly sonic modes propagating upstream in mean swirling duct flow. These eigenmodes are pressure dominated, and are analogous to acoustic modes in nonswirling mean flow. For a given frequency and azimuthal order, there are a finite number of propagating (cut-on) modes and an infinite number of evanescent (cut-off) modes. In order to determine the eigenvalues and eigenfunctions, the coupled acousticvorticity equations, (6), (7), governing the flow must be solved numerically. This is carried out using a Chebyshev spectral collocation method as described in Cooper \& Peake (2001).

In order to calculate the upstream noise in the duct, the unknown duct azimuthal orders, $\hat{m}$, and the pressure coefficients $\hat{p}_{\hat{m} s}$ in (88) must be determined. The pressure field in (87) is expressed in terms of the outer duct coordinates and matched to the inner limit of the outer pressure field (i.e. the limit of (88) as $x \rightarrow x_{s}$ ). By comparing powers of $\mathrm{e}^{\mathrm{i} \theta}$, the azimuthal mode number $\hat{m}$ for the acoustic field is found to be related to the cascade wave index $n$ by

$$
\hat{m}=m-n V,
$$

so that each plane wave mode $(n)$ produced by the cascade corresponds to an azimuthal order $(\hat{m})$ in the duct, and only a finite number of azimuthal orders appear far upstream in the duct. Equation (89) is the Tyler-Sofrin (1962) condition. The remaining terms in the pressure fields give the following equation for the modal amplitudes:

$$
\begin{aligned}
& \sum_{s=1}^{\infty} \hat{p}_{\hat{m} s} \mathscr{L}_{\hat{m} s}(r) \exp \left\{\mathrm{i} k_{\hat{m} s}^{-} x_{s}\right\}=\mathrm{i} \rho_{0} M_{\infty} c_{0} \exp \left(\mathrm{i} m k(r) x_{s}\right) \exp (-\mathrm{i} K \delta(\operatorname{Re}[F(-\infty)] \\
& \left.\left.\quad+k_{n} \operatorname{Im}[F(-\infty)]\right)\right) R_{n}(r)\left[\frac{K}{\beta_{\infty}^{2}}+\sigma_{n}\right] \exp \left(-\mathrm{i}\left(\sigma_{n}+K \frac{M_{\infty}^{2}}{\beta_{\infty}^{2}}\right) \delta \operatorname{Re}[F(-\infty)]\right. \\
& \left.\quad-\mathrm{i} \eta_{n} \delta \operatorname{Im}[F(-\infty)]\right) \equiv f_{n}(r),
\end{aligned}
$$

which can be solved for each value of $n$. The radial dependence of the cascade radiation is accounted for by summing over all duct radial orders, $s$. The duct modal amplitudes $\hat{p}_{\hat{m} s}$ can be determined by multiplying (90) by $r \mathscr{L}_{\hat{m} s}^{\dagger}(r)$ and integrating across the duct radius (with $\dagger$ denoting the complex conjugate). This generates a 
matrix equation of the form

$$
\boldsymbol{H P}=\boldsymbol{Q},
$$

where $\boldsymbol{P}=\left[\hat{p}_{\hat{m} 1} \exp \left(\mathrm{i} k_{\hat{m} 1}^{-} x_{s}\right), \hat{p}_{\hat{m} 2} \exp \left(\mathrm{i} k_{\hat{m} 2}^{-} x_{s}\right), \ldots\right]$ and

$$
H_{q s}=\int_{r_{h}}^{r_{t}} \mathscr{L}_{\hat{m} q}^{\dagger}(r) \mathscr{L}_{\hat{m} s}(r) r \mathrm{~d} r, \quad Q_{q}=\int_{r_{h}}^{r_{t}} \mathscr{L}_{\hat{m} q}^{\dagger}(r) f_{n}(r) r \mathrm{~d} r .
$$

In order to solve (91), the system of equations must be truncated, and this is carried out by including all upstream propagating cut-on duct modes and a sufficient number of cut-off modes to ensure convergence. (Note that, in the absence of swirl, the eigenfunctions are orthogonal, rendering $\boldsymbol{H}$ diagonal and the modal amplitudes can be extracted exactly.) Ultimately, only the modal amplitudes associated with the duct cut-on modes are of interest since it is these which contribute to the acoustic field far upstream.

In summary, the gust-cascade interaction determines the amplitudes of the waves which can propagate away from the cascade at each radius. These cut-on waves are then used to reconstruct the acoustic field in the three-dimensional swirling duct flow upstream of the stator. The matching of pressure fields between inner and outer regions handles the way in which the cascade cut-on condition differs from that in an annulus. The cascade cut-on condition determines which cascade modes propagate just upstream of the cascade, while the annular cut-on condition determines which parts of the radiation escaping the cascade propagate upstream in three dimensions.

\section{Results}

Each harmonic of the initial wake gives rise to an incident gust with frequency $\omega=m \Omega_{F}$ and corresponds to a blade passing frequency (or BPF) tone. All results will be expressed in terms of these frequencies. At each frequency, the upstream-radiated sound field produced by the wake-stator interaction is comprised of a number of azimuthal orders, $\hat{m}$, determined by (89). A measure of the radiated sound at each $\mathrm{BPF}$ is the total cross-sectionally averaged pressure field given by

$$
\begin{aligned}
P_{m} & =\int_{0}^{2 \pi} \int_{r_{h}}^{1}\left|p_{m}(x, r, \theta)\right|^{2} r \mathrm{~d} r \mathrm{~d} \theta, \\
& =2 \pi \sum_{\hat{m}=-\infty}^{\infty} \sum_{s=1}^{s^{\prime}} \sum_{t=1}^{s^{\prime}} \hat{p}_{\hat{m} s} \hat{p}_{\hat{m} t}^{\dagger} \exp \left(\mathrm{i}\left(k_{\hat{m} s}^{-}-k_{\hat{m} t}^{-}\right) x\right) \int_{r_{h}}^{1} \mathscr{L}_{\hat{m} s}(r) \mathscr{L}_{\hat{m} t}^{\dagger}(r) r \mathrm{~d} r,
\end{aligned}
$$

where $p_{m}$ is the local pressure at frequency $m \Omega_{F}$ and $s^{\prime}$ is the number of cut-on modes for each value of $\hat{m}$.

The same mean flow and initial wake conditions as used in $\S 2.4$ were used to generate subsequent results. The number of stator vanes was taken to be $V=50$, each vane has a profile given by a NACA four-digit section and the ratio of length scales $b^{*} / r_{t}^{*}=0.1$.

First, the effect of blade geometry on the upstream-radiated noise is assessed. In figure 4, uncambered NACA $00 \mathrm{XX}$ blades with varying angle of attack for a range of thicknesses are considered, where XX denotes the maximum blade thickness as a percentage of the blade chord. The rotor-stator spacing is taken to be $x_{s}=0.5$. Significant variation is observed both with blade thickness and angle of attack. The noise field is dominated by $2 \mathrm{BPF}$ results, even though the largest amplitude of the incident gust corresponds to the first harmonic (1BPF). This is partly because only 
(a)

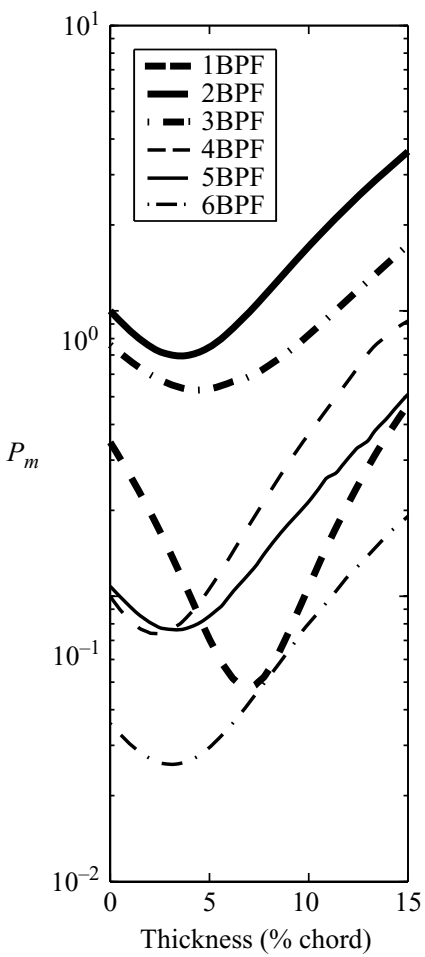

(b)

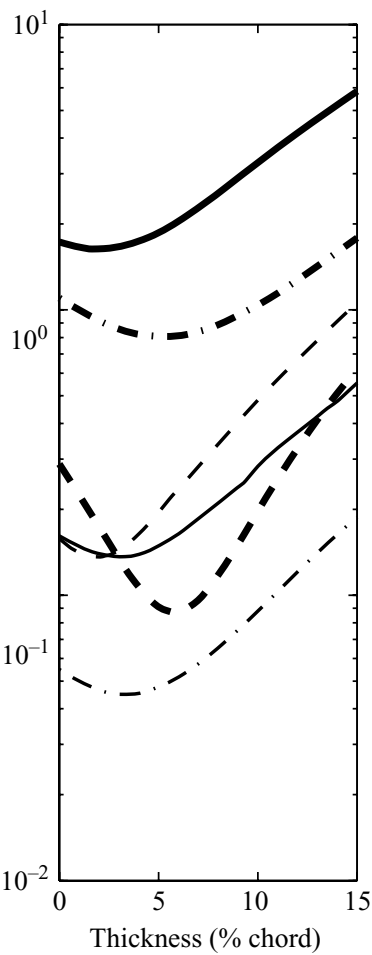

(c)

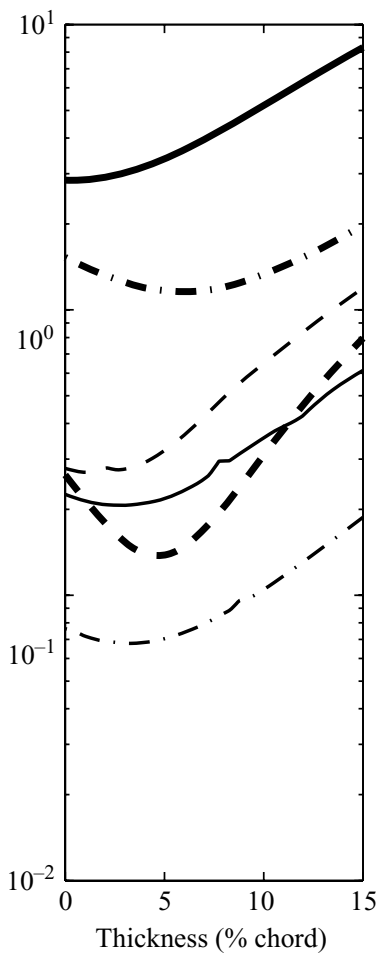

FIGURE 4. Variation of total pressure field, $P_{m}(x=0)$, with blade thickness at different angles of attack, $l_{\infty}$, where $x_{s}=0.5$ and blade camber $=0$. (a) $l_{\infty}=0^{\circ},(b) l_{\infty}=4^{\circ}$, (c) $l_{\infty}=8^{\circ}$.

one plane-wave mode is cut on by the cascade interaction at the first harmonic frequency, whereas the second harmonic consists of two plane-wave modes which are cut on over a larger proportion of the duct radius. In general, there is a greater tendency for cascade modes to be cut on near the tip than the hub, and more modes become cut on at higher frequencies. The acoustic response is then dependent on both the amplitude of the incident wave and the extent to which cascade modes are cut on by interaction with the stator. These results show that the ability to investigate a large number of blade-passing frequencies with this model enables the largest contributions to the sound field to be identified. Figure 5 shows the effects of camber for differing angles of attack, for a blade thickness of $12 \%$, again with $x_{s}=0.5$. There is strong variation with camber for the first two harmonics where the results show a well-defined minimum in each case. A minimum response is also evident for all harmonics in the thickness results of figure 4 . The cascade response is governed largely by the factor $R_{n}(r)$ in (81) and is comprised of a number of terms which describe the generation of upstream-radiated acoustic waves. The influence of blade geometry is contained in directivity functions associated with specific aspects of the geometry. In addition to this, there are several phase terms which account for the distortion of waves close to the cascade and the reflection of waves by other blade surfaces before scattering away from the cascade. However, the minimum response does not appear to be attributable to one particular geometry term; rather it would appear to be a complicated interaction of all these effects which leads to the overall response. Elhadidi \& Atassi (2003) have also found that sound levels can be affected 
(a)

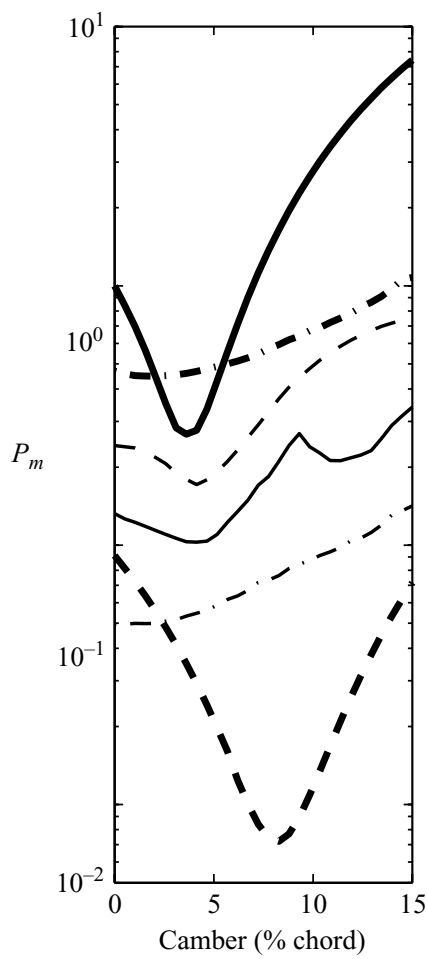

(b)

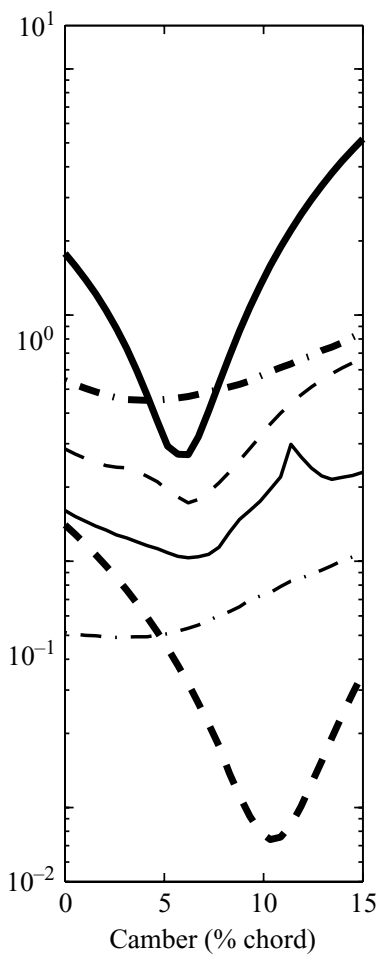

(c)

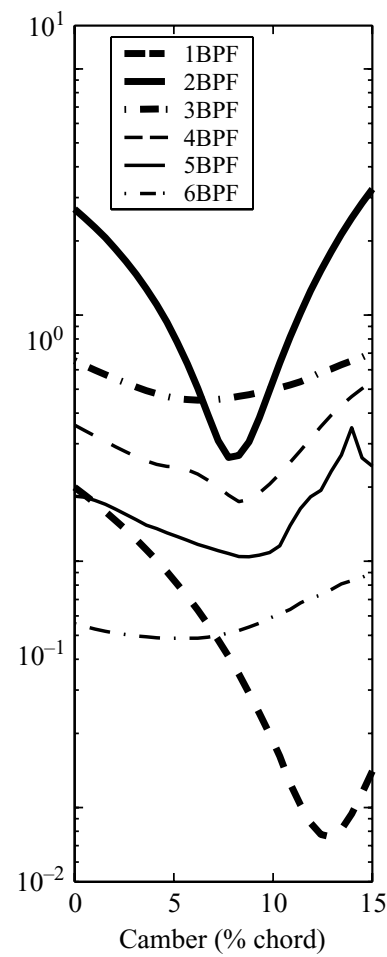

Figure 5. Variation of total pressure field, $P_{m}(x=0)$, with blade camber at different angles of attack, $l_{\infty}$, where $x_{s}=0.5$ and blade thickness $=12 \%$. (a) $l_{\infty}=0^{\circ}$, (b) $l_{\infty}=4^{\circ}$, (c) $l_{\infty}=8^{\circ}$.

significantly by blade design and in particular that increasing blade camber generally leads to an increase in the upstream acoustic pressure.

The impact of rotor-stator gap on the noise generated is demonstrated in figure 6 . This shows that each harmonic no longer contributes to the total pressure field beyond a critical separation, and this is related directly to the plane-wave modes from the cascade interaction becoming cut off. The plane wave cut-on condition specifies that the term inside the square root in (82) and (83) must be positive, and the only parameter which varies with $x_{s}$ in this condition is $w^{2}$ through its dependence on the spanwise wavenumber $k_{3}$. Therefore, when $k_{3}$ (equivalently $x_{s}$ ) exceeds a critical value, the associated plane-wave mode becomes cut off, and this effect is demonstrated in figure 6 by sharp changes in gradient. The inset in figure 6 shows contours of $w$ as a function of radius and rotor-stator separation (for clarity, only contours where $w^{2}>0$ are shown). This shows how the cascade cut-on region shrinks as the rotor-stator gap is increased and that no noise is radiated upstream beyond $x_{s} \approx 0.71$, where $w^{2}<0$ across the whole annulus. Also plotted is the specific cut-on boundary determined by the cascade response for the BPF tone. This type of calculation demonstrates general trends very effectively and can qualitatively identify optimum conditions for reducing noise levels. The effect of increasing the rotor-stator separation on the radiated noise is related directly to the development of the radial wavenumber as the wake moves downstream. As the rotor-stator gap is increased, the wake becomes more skewed and the number of wake intersections with each stator vane increases. The correlation between number of wake intersections and noise levels has been observed by Envia \& Nallasamy (1999), 


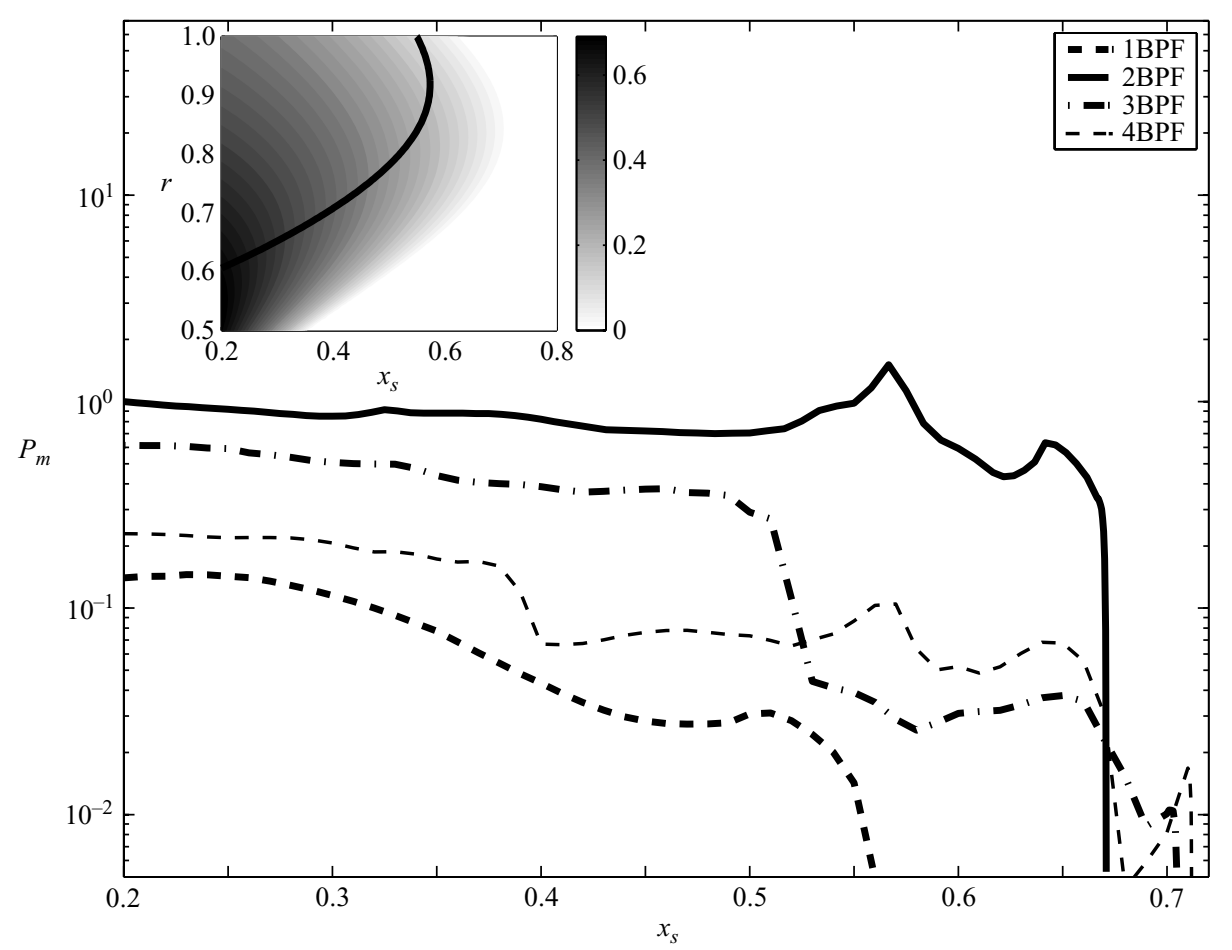

FIGURE 6. Variation of total pressure field, $P_{m}(x=0)$, with rotor-stator separation, $x_{s}$. The stator blade profile has $5 \%$ thickness, angle of attack $4^{\circ}$ and zero camber. Inset shows contours of reduced frequency parameter $w$ as a function of radius and rotor-stator separation. The solid line shows the boundary of the cut-on region for the 1BPF tone. All upstream plane-wave modes are cut off in the unshaded region.

(a)

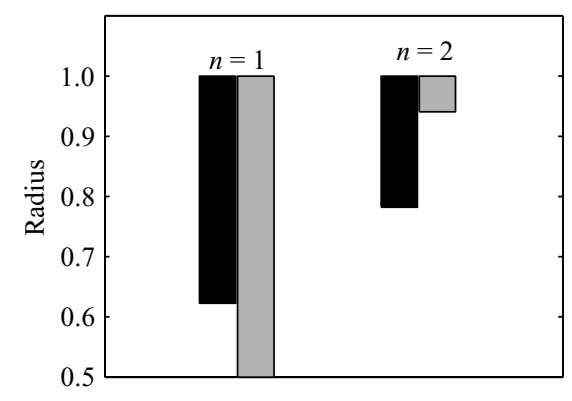

(b)

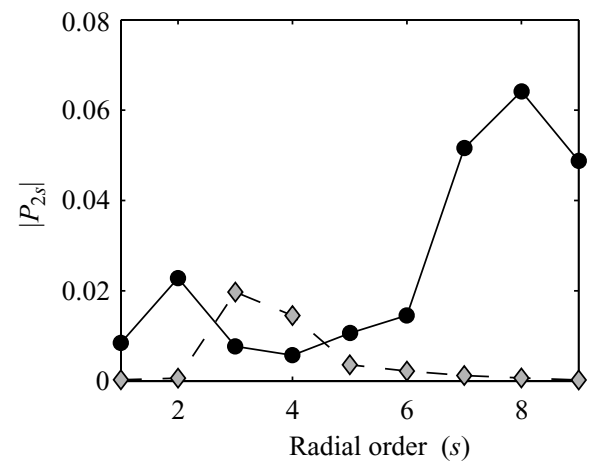

FIGURE 7. (a) Proportion of span for which the cascade radiation modes $n=1$ and $n=2$, generated at 2BPF, are cut on. Black regions denote the cut-on range with swirl included, grey regions denote the no-swirl range. (b) Amplitudes of the pressure coefficients $\hat{p}_{\hat{m} s}$ for $\hat{m}=2$, with swirl (black circles) and without swirl (grey diamonds).

and Elhadidi \& Atassi (2002) also concluded that spanwise modulation of the incident disturbance can lead to a reduction in the acoustic sound propagation.

In order to demonstrate the significance of including swirl, several comparisons with a non-swirling flow have been carried out. Figure 7(a) compares the extent to 
(a)

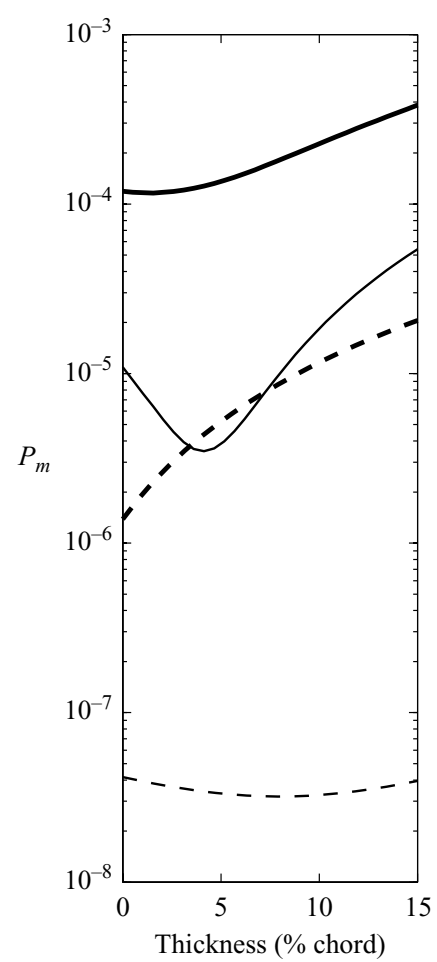

(b)

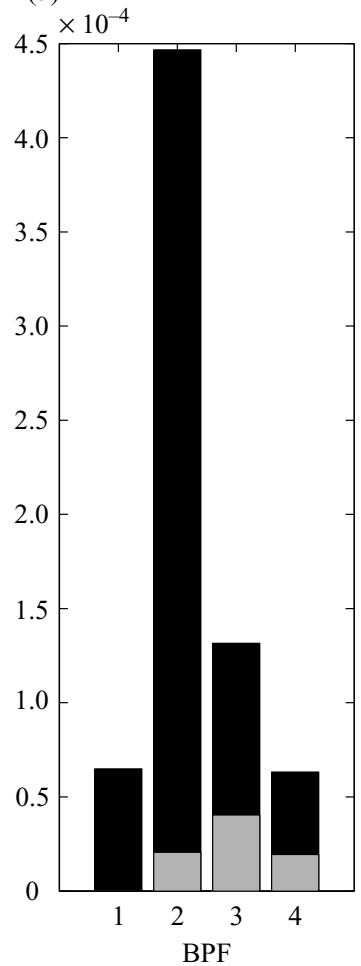

FiguRE 8. (a) Variation of total pressure field, $P_{m}(x=0)$, with blade thickness (angle of attack $l_{\infty}=4^{\circ}$ ) at 2BPF. Solid lines are the contribution from $\hat{m}=2$, and dashed lines are the contribution from $\hat{m}=-48$. The bold lines are the results with swirl, thin lines are the no-swirl results. $(b)$ Total pressure field $P_{m}(x=0)$ for $m=1, \ldots, 4$ with swirl included (black bars) and without swirl (grey bars), (thickness $=15 \%, l_{\infty}=4^{\circ}$ ).

which the cascade mode orders $n=1$ and $n=2$ at the second harmonic frequency are cut on (these generate the azimuthal mode orders $\hat{m}=2$ and $\hat{m}=-48$ of the dominant $2 \mathrm{BPF}$ tone). Swirl is seen to have a large influence in both cases, with the $n=1$ mode cut on over a smaller range of radii, and the $n=2$ mode cut on over a larger proportion of the span than in the absence of swirl. This effect can be related to the orientation of the waves relative to the swirl. It is known that in the duct, azimuthal modes co-rotating with the swirl $(\hat{m}>0)$ tend to be cut off by swirl, and those which are counter-rotating $(\hat{m}<0)$ tend to be cut on by swirl. In the above example, the $n=1$ cascade mode corresponds to a co-rotating duct mode, and the $n=2$ cascade mode corresponds to a counter-rotating duct mode. Figure $7(b)$ compares the duct pressure coefficients, $\hat{p}_{\hat{m} s}$, determined from (90), when $\hat{m}=2$. Again, significant differences arise with the inclusion of swirl with the order, $s$, of the dominant radial mode (in this case) increasing when swirl is included. Figure 8 considers how swirl affects the final measure of noise, by comparing the values of the total averaged pressure field $P_{m}(x=0)$. In figure $8(a)$, the relative contributions to the dominant $2 \mathrm{BPF}$ mode (from $\hat{m}=2$ and $\hat{m}=-48$ ) are compared. In both cases, the levels predicted when swirl is not included are significantly less than when the swirl is accounted for. In figure $8(b)$, results are presented for the first four harmonic frequencies. Noise levels predicted in the absence of swirl all lie far below the swirling 
flow results. Of particular interest is the prediction of zero upstream-radiated noise at $1 \mathrm{BPF}(\hat{m}=-24)$. This is because there are no acoustic duct modes cut on in the absence of swirl and so no contribution to the far-field noise, whereas there are three cut-on modes which contribute to the noise when swirl is included. A second feature is that, in the absence of swirl, the largest contribution comes from the $3 \mathrm{BPF}$ response.

These results show that the effects of swirl influence the whole of the rotor-stator interaction process. Swirl affects the wake evolution, bringing about the wake-skewing, which then determines the nature of the waves impinging on the cascade. The input to the cascade determines the acoustic response and the cut-on conditions of the radiated modes. The cut-on conditions for the duct acoustic modes are also affected heavily by swirl. The final measure of the noise, the total averaged pressure field, is affected through the matching of the two pressure fields and by the changes to the duct eigenmodes.

\section{Asymptotic approximation to radiated acoustic field}

The determination of eigenvalues and eigenvectors and the calculation of the radiated pressure field in swirling flow involves considerable numerical evaluation for each harmonic frequency. In a similar manner to that used for the evolution of the rotor wake, asymptotic techniques can be applied to the equations governing the pressure-dominated modes since, in general, the azimuthal order $(\hat{m})$ of these modes is large. The asymptotic structure of these modes will be described in $\S 5.1$. Further asymptotic techniques can be applied to the integrals in (92), which allows the dominant contributions to the sound field, together with trends and scaling laws to be identified, and this will be described in $\S 5.2$.

\subsection{Large $\hat{m}$ description of radiation modes}

Following the suggestion of Envia (1998), an asymptotic approximation for the nearly sonic modes using the WKB method is now developed. The vortical and potential parts of the velocity field can be written as the modal decompositions

$$
\begin{gathered}
\boldsymbol{u}(x, r, \theta, t)=\sum_{s=1}^{\infty} \boldsymbol{A}_{\hat{m} s}(r) \exp \left(\mathrm{i}\left\{\hat{m} k_{\hat{m} s}^{-} x+\hat{m} \theta-\omega t\right\}\right), \\
\Phi(x, r, \theta, t)=\sum_{s=1}^{\infty} \phi_{\hat{m} s}(r) \exp \left(\mathrm{i}\left\{\hat{m} k_{\hat{m} s}^{-} x+\hat{m} \theta-\omega t\right\}\right),
\end{gathered}
$$

where $\omega=m \Omega_{F}$. The modal amplitudes $\boldsymbol{A}_{\hat{m} s}, \phi_{\hat{m} s}$ are taken to be $O(1)$, so that the leading-order contribution to the total unsteady velocity field comes from $\nabla \Phi$. Under the assumption of large $\hat{m}$, the equation governing the potential becomes, to leading order,

$$
\frac{\mathrm{d}^{2} \phi_{\hat{m} s}}{\mathrm{~d} r^{2}}=-\hat{m}^{2} f(r) \phi_{\hat{m} s},
$$

where $f=\left(\Lambda_{\hat{m} s}^{2} / c_{0}^{2}-1 / r^{2}-k_{\hat{m} s}^{-2}\right)$, with $\Lambda_{\hat{m} s}=k_{\hat{m} s}^{-} U-m \Omega_{F} / \hat{m}+W / r$. Note that the pressure eigenfunction in $\S 3$ is given by $\mathscr{L}_{\hat{m} s}=-\mathrm{i} \rho_{0} \Lambda_{\hat{m} s} \phi_{\hat{m} s}$.

The solution to (97) depends on the sign of $f$, and the possibility that there exist turning points, where $f=0$, needs to be considered. For example, suppose that $f<0$ for $r_{h} \leqslant r<c$ and $f>0$ for $c<r \leqslant 1$, with $f(c)=0$. Then, away from the turning 
point, the potential is given by the WKB solution

$$
\begin{array}{ll}
\phi_{\hat{m} s}=\frac{\alpha_{1}}{\hat{m}^{1 / 2}(-f)^{1 / 4}}\left\{\exp \left(\hat{m} w_{1}(r)\right)+\exp \left(-\hat{m} w_{1}(r)\right)\right\}, & r_{h} \leqslant r<c, \\
\phi_{\hat{m} s}=\frac{\gamma_{1}}{\hat{m}^{1 / 2}(f)^{1 / 4}}\left\{\exp \left(\mathrm{i} \hat{m} w_{2}(r)\right)+\exp \left(-\mathrm{i} \hat{m} w_{2}(r)\right)\right\}, & c<r \leqslant 1,
\end{array}
$$

where $\alpha_{1}$ and $\gamma_{1}$ are constants and

$$
w_{1}(r)=\int_{r_{h}}^{r} \sqrt{-f(\xi)} \mathrm{d} \xi, \quad w_{2}(r)=\int_{r}^{1} \sqrt{f(\xi)} \mathrm{d} \xi .
$$

The solutions in equations (98) and (99) satisfy the leading-order boundary conditions $\mathrm{d} \phi_{\hat{m} s} / \mathrm{d} r=0$ at $r=r_{h}, 1$.

In the vicinity of the turning point, using the substitution $y=r-c$, (97) becomes Airy's equation

which has solution

$$
\frac{\mathrm{d}^{2} \phi_{\hat{m} s}}{\mathrm{~d} y^{2}}+\hat{m}^{2} f^{\prime}(c) y \phi_{\hat{m} s}=0
$$

$$
\phi_{\hat{m} s}=A \operatorname{Ai}\left(-\hat{m}^{2 / 3} f^{\prime}(c)^{1 / 3} y\right)+B \operatorname{Bi}\left(-\hat{m}^{2 / 3} f^{\prime}(c)^{1 / 3} y\right),
$$

with $A$ and $B$ constants to be determined. The unknown eigenvalues, $k_{\hat{m} s}^{-}$, and the constants in each of the three solution regions can be identified using matched asymptotic expansions. The eigenvalues are then determined from the condition

$$
2 \hat{m} w_{2}(c)-\left(2 s+\frac{1}{2}\right) \pi+2 \psi=0, \quad s=0,1,2, \ldots
$$

where $\tan \psi=\exp \left\{-2 \hat{m} w_{1}(c)\right\} / 2$. The integer $n$ labels the infinite spectrum of axial eigenvalues for a given $\hat{m}$, and corresponds to the usual radial order. Similar results can be obtained if $f>0$ for $r_{h} \leqslant r<c$ and $f<0$ for $c<r \leqslant 1$.

The eigenvalues and corresponding eigenfunctions have been determined using the above asymptotics for $\hat{m}=-48$ and, in order to test the range of validity of the large- $\hat{m}$ assumption, for $\hat{m}=2$. Results are plotted in figure 9, and good agreement is obtained. For $\hat{m}=-48$, the eigenmode shown in figure $9(b)$ has a turning point at $r \approx 0.584$, while for $\hat{m}=2$ there is no turning point, and the WKB approximation to the eigenfunction takes the oscillatory form (99) everywhere between the hub and the tip.

A useful way of visualizing the mode structure is to consider the ray paths. In the oscillatory part of the WKB approximation (99), the mode is written as the sum of components $\exp \left(\mathrm{i} \Theta_{ \pm}\right)$, where the phase is

$$
\Theta_{ \pm}=\hat{m} k_{\hat{m} s}^{-} x+\hat{m} \theta \pm \hat{m} w_{2}(r),
$$

so that for each point in space where the solution is oscillatory there are two ray directions, parallel to $\nabla \Theta_{ \pm}$. These rays propagate along the duct in a helical manner. For cases in which the inner or outer (rigid) wall lies in an oscillatory region, the rays are reflected from that wall, with for instance at the outer wall, the radially outward-going ray associated with $\nabla \Theta_{+}$being reflected into the radially inward-going ray associated with $\nabla \Theta_{-}$. At a turning point, $r=c$ say, it is easy to check that the radial component of $\nabla \Theta_{ \pm}$is zero, corresponding to the circle $r=c$ being the circular ray envelope, or caustic.

In the case of zero swirl and uniform axial flow, as studied by Chapman (1994), if a caustic is present between the inner and outer walls, then it must necessarily 
(a)

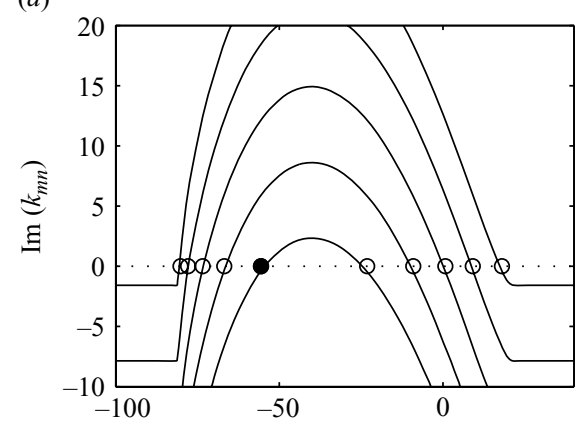

(c)

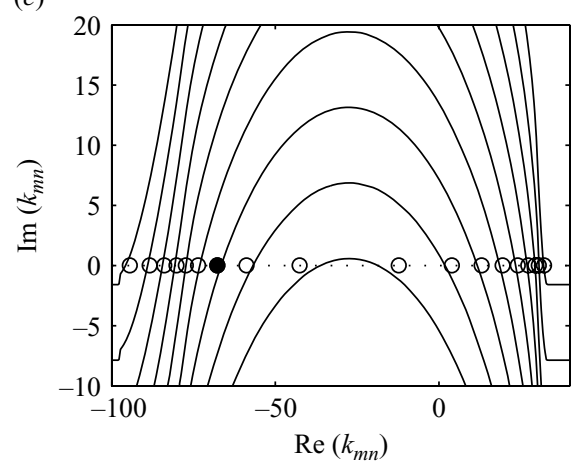

(b)

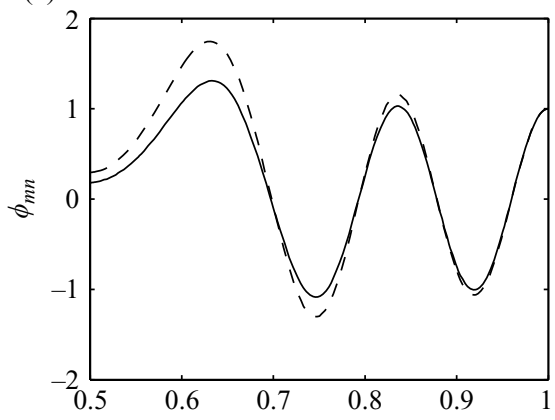

(d)

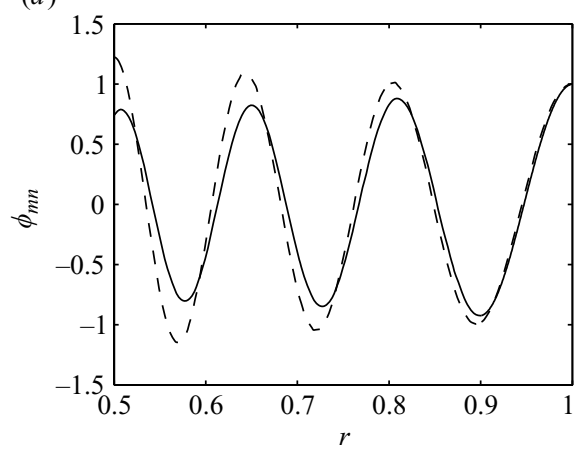

FIGURE 9. Asymptotic approximation for acoustic modes in swirling flow. (a) Eigenvalues for $\hat{m}=-48$. Circles denote numerical values and the lines plot the function on the left-hand side of (103) for different values of $s$. The asymptotic approximation corresponds to the point where the lines cross zero. $(b)$ Eigenfunction for the $(s=4)$ eigenmode denoted by the filled circle in $(a)$; - , asymptotic approximation; ---, numerical solution. (c) As for $(a)$ but with $\hat{m}=2$. (d) As for $(b)$ but with $\hat{m}=2, s=6$.

delineate an oscillatory region outboard, which extends all the way to the outer wall, from a 'quiet' region inboard where there are no rays. In the quiet region, the WKB solution takes the real-exponential form (98). In the oscillatory region, the individual ray paths are straight lines, which, on reflection by the outer wall, form what is termed by Chapman a 'piecewise linear helix' winding along the duct. However, when swirl and radially varying mean axial flow are considered, a richer variety of behaviour can be found. Four such possibilities are plotted in figure 10, where the projections of the ray directions onto planes of constant $x$ (i.e. directions given by the radial and tangential components of $\nabla \Theta_{ \pm}$) are plotted. In each of these, the rays are no longer straight lines, owing to the radial variation of the mean flow. In figure 10(a) $(\hat{m}=-48, s=0)$, there are, in fact, two turning points between the inner and outer walls, and the rays are constrained to lie between these two radii without ever reaching the walls. In figure $10(b)(\hat{m}=-48, s=4)$, there is a single turning point, and the rays form a pattern analogous to the Chapman case, with a single inner quiet zone and rays reflected off the outer wall. In figure $10(c)(\hat{m}=2, s=3)$, again there is a single turning point between the inner and outer walls, but now the quiet zone lies outside the single caustic cylinder, while in figure $10(d)(\hat{m}=2, s=7)$, there is no turning point between the inner and outer walls and the rays propagate through the whole of the annulus. 
(a)

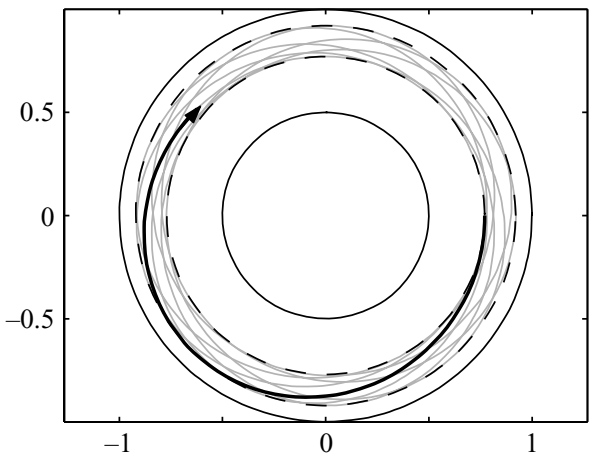

(c)

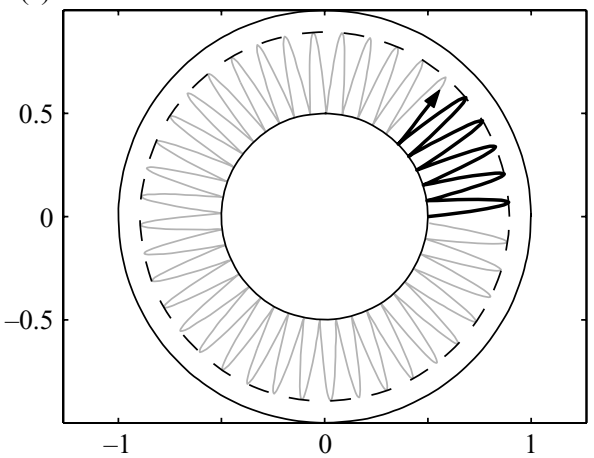

(b)

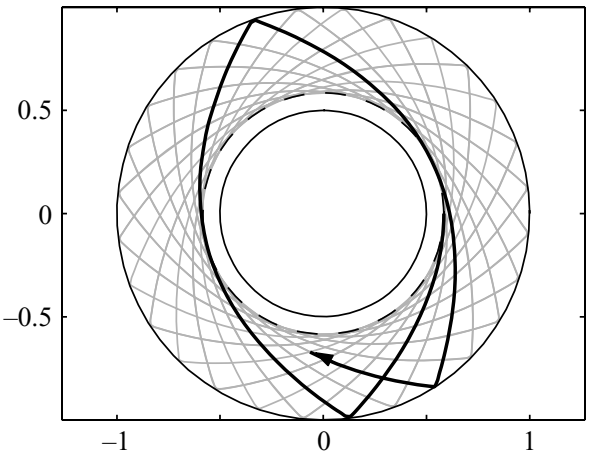

(d)

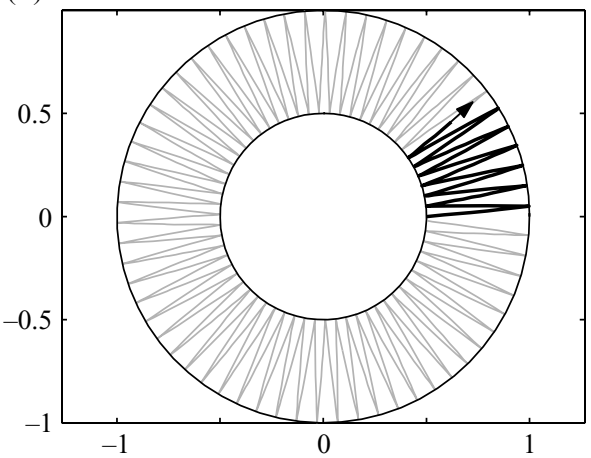

FIGURE 10. Projection of ray paths onto planes of constant $x$ for acoustic modes in swirling flow. Dashed lines denote ray caustics which delineate oscillatory regions from 'quiet' regions. (a) $\hat{m}=-48, s=0,(b) \hat{m}=-48, s=4,(c) \hat{m}=2, s=3,(d) \hat{m}=2, s=7$.

It is clear that the sign of $f(r)$ determines whether or not real rays are found at a given radius. In the Chapman (1994) case of $U(r)=U_{0}$ and zero swirl, it follows that $f(r)$ is a monotonically increasing function of $r$. The oscillatory WKB solution (99) can then only occur if

$$
f(\infty) \equiv \frac{\left(k U_{0}-m \Omega_{F} / \hat{m}\right)^{2}}{C_{0}^{2}}-k^{2}>0,
$$

and if this is the case there must then be a single caustic located at a finite value of $r$. The condition $f(\infty)>0$ corresponds to the condition for a plane sound wave with axial wavenumber $k$ to be able to propagate in the uniform axial flow, and is exactly what is to be expected since, as $r \rightarrow \infty$, the effects of the azimuthal wavenumber $\hat{m}$ must disappear.

When $W \neq 0$ and $U=U(r)$, a more complicated variation of $f(r)$ emerges since it is no longer necessary for $f$ to be a monotonic function of $r$. In this example, the logarithmic term introduced into the expression for $U(r)$ in order to enforce uniform stagnation enthalpy only makes sense in a strictly annular duct. However, it can be seen that $f \rightarrow+\infty$ as $r \rightarrow 0$, while for $r \neq 0, f$ may change sign a number of times. In the case shown in figure $10(a), f(1)<0$ and there are two caustics (lying within the annulus) with rays confined away from the walls. This sort of structure arises from the combination of the swirl and a radially dependent axial flow. 
(a)

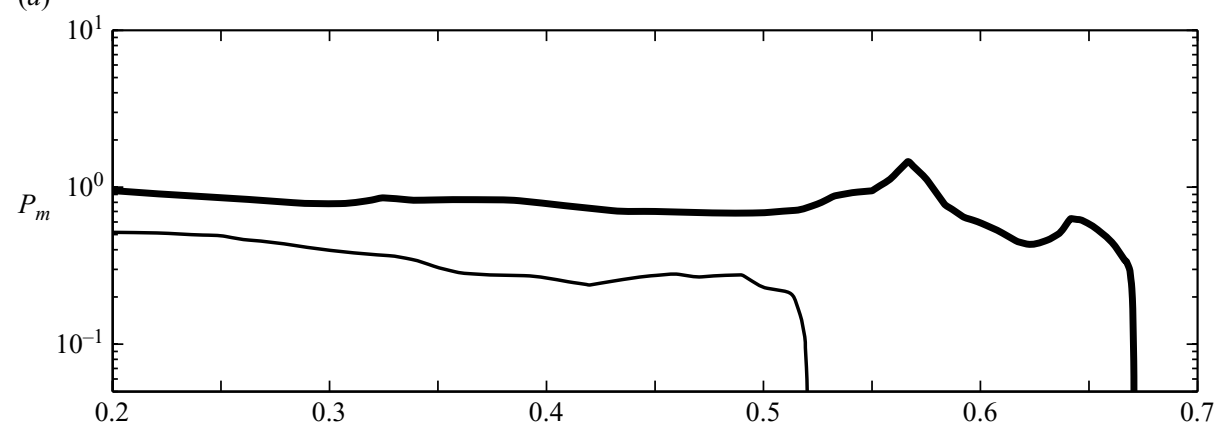

(b)

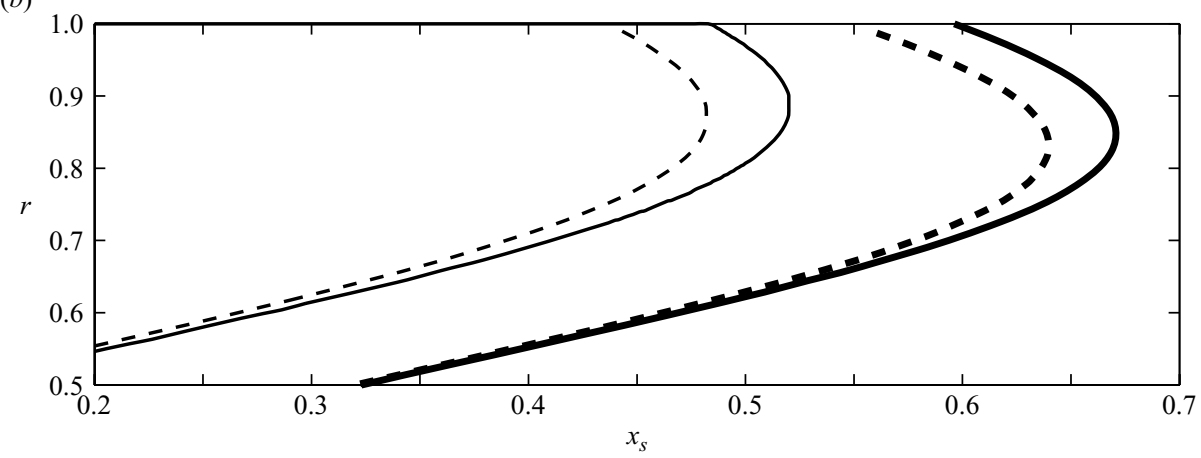

FIgURE 11. (a) Dominant contributions to total pressure field at 2BPF (bold line, $\hat{m}=2$ ) and $3 \mathrm{BPF}$ (thin line, $\hat{m}=28$ ) as rotor-stator separation is increased. (b) Solid lines shows cascade cut-on boundaries as a function of rotor-stator separation and dashed lines show stationary-phase points for the highest radial-order acoustic mode.

\subsection{Dominant contribution to modal amplitudes in rotor-stator interaction calculation}

This section deals with approximating the integrals in (92). In cases in which the eigenmode $\mathscr{L}_{\hat{m} q}(r, x)$ is oscillatory, i.e. takes the propagating-ray form (99), the integral $Q_{q}$ can be approximated using the method of stationary phase when $\hat{m}$ and $m$ are large. It is easy to see that such a stationary-phase point satisfies $m k_{r} x_{s}= \pm \sqrt{f}$, corresponding to the case in which the radial phase speed of the incident wake disturbance matches the radial phase speed of either the radially outward or inward wave in the acoustic eigenfunction. If there exists a single stationary-phase point within the annulus then $Q_{q}=O(1 / \hat{m})$, while if no stationary points exist then $Q_{q}$ can be approximated using integration by parts to yield $Q_{q}=O\left(1 / \hat{m}^{3 / 2}\right)$. Note also that the stationary phase analysis can also be completed in cases when $\hat{m}$ is not large, as long as the incident azimuthal order $m$ is large. In this case, the right-hand side of the WKB equation (97) is $O\left(\mathrm{~m}^{2}\right)$.

This analysis can be used to explain the behaviour in figure 6 , where, as the rotorstator gap is increased, the total acoustic pressure response generally increases before falling rapidly to zero. With increasing rotor-stator separation, the incident wake becomes increasingly distorted and the response for each $\hat{m}$ becomes dominated by the behaviour of the highest radial-order acoustic mode. Stationary-phase points have been calculated for the highest radial order when $\hat{m}=2$ (the dominant contribution to the $2 \mathrm{BPF}$ response) and $\hat{m}=28$ (the dominant contribution to the $3 \mathrm{BPF}$ response) and these are plotted in figure 11 together with the cut-on boundaries determined by 
the cascade response. When $\hat{m}=2$, the acoustic pressure response shows an increase with the onset of a second stationary-phase point at the tip, at $x_{s} \approx 0.55$. The two stationary-phase points then approach each other on the radius as $x_{s}$ is increased further, leading to a reduction in noise as their two contributions interfere with each other. Once the two stationary points approach very close to each other, the noise rises again, and there is a peak around $x_{s} \approx 0.64$ close to the point of coalescence. The presence of this peak is explained mathematically by noting that the close proximity and coalescence of two stationary-phase points is described by an Airy function of the form $\mathrm{Ai}\left(-\hat{m}^{2 / 3} R\right)$, where $R$ is proportional to the radial separation of the two stationary-phase points. This Airy function then has its maximum value at $\hat{m}^{2 / 3} R \approx 1$, i.e. for large $\hat{m}$ just before the stationary-phase points coalesce.

Once the stationary points have coalesced they move into the complex $r$-plane, and $Q_{q}$ is then approximated by integration by parts to yield a smaller result. In addition to this, the portion of the radius over which the local cascade emits cut-on radiation shrinks, resulting in a rapid fall in the pressure response which becomes zero when the rotor-stator separation lies beyond the cascade cut-on region $\left(x_{s} \approx 0.67\right)$. Similar behaviour can be seen in figure 11 for $\hat{m}=28$. In some other cases, not shown, the acoustic response can increase for $x_{s}$ beyond the location where the two stationaryphase points coalesce, and this can be attributed to the size of the local cascade radiation, which is known to increase sharply close to cut-off.

\section{Summary}

An analytically based model has been developed to study and predict rotor-stator interaction noise in aeroengines, in particular upstream-radiated noise. The threedimensional cylindrical duct geometry used to represent the aeroengine is retained throughout and coupled to a local linear cascade model for the stator.

An asymptotic analysis which exploits the large rotor blade number is used to determine the downstream evolution of the rotor wake in mean swirling flow. In the presence of swirling flow, the wake becomes distorted by centrifugal and Coriolis forces as it propagates downstream, and under this asymptotic treatment the equations governing the wake evolution can be simplified in the main body of the flow to three coupled first-order differential equations in $x$, where $r$ appears only as a parameter. Close to the boundaries, a correction to this solution is required in order to satisfy the flow boundary conditions. It is shown that the wake becomes increasingly skewed as it propagates downstream and the degree of distortion is governed by the development of a radial wavenumber, $x m k_{r}(r)$, so that $k_{r}(r)$ determines the extent of rotation at each radius.

Once the wake is evolved as far as the stator, a cascade calculation determines the amount of forward radiation produced by each incident harmonic wave. The cascade response is formulated in terms of plane-wave modes and this information can be used to determine the upstream-radiated pressure field across the whole annulus just ahead of the stator. The effects of the mean swirl on the cascade response have been included asymptotically, and, in particular, it has been shown how the non-swirling results of Evers \& Peake (2002) can be applied here as well. The cascade pressure field is then reconstructed in terms of cylindrical duct modes in mean swirling flow. The matching of pressure fields handles the way in which the cascade cut-on condition differs from that in an annulus; the cascade cut-on condition determines which cascade modes propagate just upstream of the cascade, while the annular cut-on condition 
determines which parts of the radiation escaping the cascade propagate upstream in three dimensions.

The cascade model allows for the effects of stator blade geometry to be investigated. Results show that for uncambered blades, thickness and angle of attack tend to increase the amount of radiated noise. For cambered blades there is significant variation across a range of blade cambers. The results also show that the largestamplitude incident wave does not necessarily produce the largest contribution to the total acoustic field. Each response depends on the relative contribution from each azimuthal order generated and this is governed in part by the number of stator blades. It is also shown that, as the rotor-stator separation is increased, each $\hat{m}$ contribution becomes cut off at critical separations determined from the analysis, leading ultimately to significant reductions in noise. In practice, it may not be possible to locate the stator at sufficiently large distances from the rotor owing to design and weight constraints. The results from this investigation suggest that an alternative to increasing the rotor separation as a means of reducing the interaction noise would be to force the wake to be more skewed initially.

Finally, asymptotic analysis on the acoustic field in swirling flow based on large azimuthal order was considered. Approximate solutions for eigenvalues and eigenvectors were determined and good agreement with numerical results was obtained. The ray structure of the acoustic modes in a mean swirling flow is found to provide an even richer variety of behaviour than the results for uniform flow studied by Chapman (1994). For instance, in some circumstances the acoustic mode is trapped between caustics away from the walls, so that application of acoustic wall lining designed to attenuate the sound would presumably be ineffective in such a case. Further asymptotic analysis indicates that dominant contributions to the total pressure field come from stationary-phase points in the integrals, and may also explain the increase in acoustic response prior to cut-off.

In summary, the analysis presented in this paper describes a scheme which allows for the systematic prediction of the effects of stator-blade geometry and swirl on forward-radiated rotor-stator interaction noise. Various asymptotic analyses are used to identify general trends in rotor wake evolution and the acoustic response.

A. J.C. acknowledges the financial support provided by The Royal Society. The authors are also very grateful to Professor E. J. Kerschen for many helpful discussions and to Dr I. Evers for providing numerical code for the cascade calculation.

\section{Appendix. Evolution equations for mean potential flow}

For a potential mean flow in a uniform duct, the leading-order terms in (6) governing the vortical part of the disturbance become

$$
\begin{array}{r}
U \frac{\partial a_{x}}{\partial x}=0, \\
U \frac{\partial a_{r}}{\partial x}-\frac{2 W}{r} a_{\theta}=0, \\
U \frac{\partial a_{\theta}}{\partial x}=0,
\end{array}
$$

which has solution

$$
a_{x}(x, r)=a_{x}(0, r),
$$




$$
\begin{aligned}
& a_{r}(x, r)=\frac{2 W x}{U r} a_{\theta}+a_{r}(0, r), \\
& a_{\theta}(x, r)=a_{\theta}(0, r),
\end{aligned}
$$

with the potential defined by (19).

A boundary-layer correction is also required in this case, but the boundary conditions can be satisfied by a correction to the potential only, which is governed by the equation

$$
\frac{\partial^{2} \hat{\phi}_{1}}{\partial R^{2}} \pm 2 \mathrm{i} x k_{r} \frac{\partial \hat{\phi}_{1}}{\partial R}-\lambda^{2} \hat{\phi}_{1}=0
$$

where \pm refers to the inner and outer boundaries, respectively. The solution is

$$
\hat{\phi}_{1}(x, R)=f(x) \exp \{-\alpha(x) R\} \quad \text { where } \quad \alpha(x)=\mp \mathrm{i} x k_{r}\left(r_{b}\right) \pm \sqrt{\frac{1}{r_{b}^{2}}+k^{2}} .
$$

The amplitude $f(x)$ is determined by the wall boundary condition, and is given by

$$
f(x)= \pm \frac{\left\{a_{r}\left(x, r_{b}\right)+\mathrm{i} x k_{r}\left(r_{b}\right) \phi_{1}\left(x, r_{b}\right)\right\}}{\alpha(x) \mp \mathrm{i} x k_{r}\left(r_{b}\right)} .
$$

\section{REFERENCES}

Atassi, H. M., Ali, A. A., Atassi, O. V. \& Vinogradov, I. V. 2004 Scattering of incident disturbances by an annular cascade in swirling flow. J. Fluid Mech. 499, 111-138.

Chapman, C. J. 1994 Sound radiation from a cylindrical duct. Part 1. Ray structure of the duct modes and of the external field. J. Fluid Mech. 281, 293-311.

Cooper, A. J. \& Peake, N. 2001 Propagation of unsteady disturbances in a slowly varying duct with mean swirling flow. J. Fluid Mech. 445, 207-234.

ElHADIDI, B. \& Atassi, H. M. 2002 High frequency sound radiation from an annular cascade in swirling flows. AIAA Paper 2002-2560.

ElHADidi, B. \& ATASSI, H. M. 2003 High frequency formulation for interaction noise in annular cascades. AIAA Paper 2003-3133.

Elhadidi, B., Atassi, H. M., Envia, E. \& Podboy, G. 2000 Evolution of rotor wake in swirling flow. AIAA Paper 2000-1991.

Envia, E. 1998 A high frequency model of cascade noise. AIAA Paper 98-2318.

Envia, E. \& Nallasamy, M. 1999 Design selection and analysis of a swept and leaned stator concept. J. Sound Vib. 228, 793-836.

Evers, I. \& Peake, N. 2002 On sound generation by the interaction between turbulence and a cascade of airfoils with non-uniform mean flow. J. Fluid Mech. 463, 25-52.

GLEGG, S. A. L. 1999 The response of a swept blade row to a three-dimensional gust. J. Sound Vib. 227, 29-64.

GolDSTEIN, M. E. 1978 Unsteady vortical and entropic distortions of potential flows round arbitrary obstacles. J. Fluid Mech. 89, 433-468.

Golubev, V. V. \& Atassi, H. M. 1996 Sound propagation in an annular duct with mean potential swirling flow. J. Sound Vib. 198, 601-616.

Golubev, V. V. \& Atassi, H. M. 1998 Acoustic-vorticity waves in swirling flows. J. Sound Vib. 209, 203-222.

Golubev, V. V. \& Atassi, H. M. $2000 a$ Unsteady swirling flows in annular cascades. Part 1: Evolution of incident disturbances. AIAA J. 38, 1142-1149.

Golubev, V. V. \& Atassi, H. M. $2000 b$ Unsteady swirling flows in annular cascades. Part 2: Aerodynamic blade response. AIAA J. 38, 1150-1158.

Hanson, D. B. 2001 Theory for broadband noise of rotor and stator cascades with inhomogeneous inflow turbulence including effects of sweep and lean. NASA/CR-2001-210762.

KocH, W. 1971 On the transmission of sound waves through a blade row. J. Sound Vib. 18, 111-128. 
Mani, R. \& Horvay, G. 1970 Sound transmission through blade rows. J. Sound Vib. 12, 59-83.

Myers, M. R. \& Kerschen, E. J. 1995 Influence of incidence angle on sound generation by airfoils interacting with high-frequency gusts. J. Fluid Mech. 292, 271-304.

Myers, M. R. \& KersChen, E. J. 1997 Influence of camber on sound generation by airfoils interacting with high-frequency gusts. J. Fluid Mech. 353, 221-259.

Peake, N. 1992 The interaction between a high-frequency gust and a blade row. J. Fluid Mech. 241, 261-289.

Peake, N. \& Kerschen, E. J. 1997 Influence of mean loading on noise generated by the interaction of gusts with a flat-plate cascade: upstream radiation. J. Fluid Mech. 347, 315-346.

Tsai, C. T. \& Kerschen, E. J. 1990 Influence of airfoil nose radius on sound generated by gust interactions. AIAA Paper 90-3912.

Tyler, J. M. \& Sofrin, T. G. 1962 Axial flow compressor noise studies. SAE Trans. 70, 309-332.

\section{CAMBRIDGE JUURNALS}

